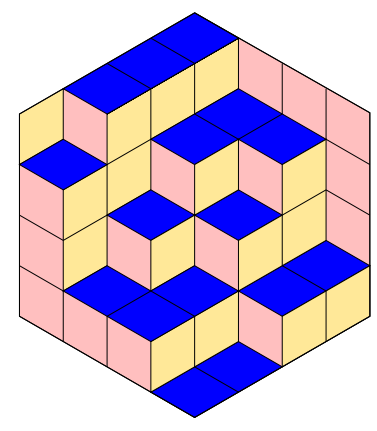

John R. Stembridge

Sign-twisted Poincaré series and odd inversions in Weyl groups

Volume 2, issue 4 (2019), p. 621-644.

<http://alco.centre-mersenne.org/item/ALCO_2019__2_4_621_0>

(C) The journal and the authors, 2019.

Some rights reserved.

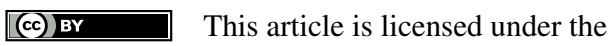

Creative Commons ATtribution 4.0 InTERnATIONAL LiCENSE.

http://creativecommons.org/licenses/by/4.0/

Access to articles published by the journal Algebraic Combinatorics on the website http://alco.centre-mersenne.org/ implies agreement with the Terms of Use (http://alco.centre-mersenne.org/legal/).

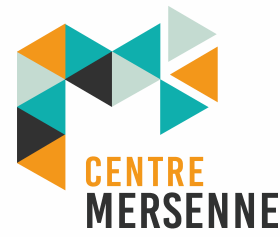

Algebraic Combinatorics is member of the Centre Mersenne for Open Scientific Publishing www.centre-mersenne.org 


\title{
Sign-twisted Poincaré series and odd inversions in Weyl groups
}

\author{
John R. Stembridge
}

\begin{abstract}
Following recent work of Brenti and Carnevale, we investigate a sign-twisted Poincaré series for finite Weyl groups $W$ that tracks "odd inversions"; i.e. the number of oddheight positive roots transformed into negative roots by each member of $W$. We prove that the series is divisible by the corresponding series for any parabolic subgroup $W_{J}$, and provide sufficient conditions for when the quotient of the two series equals the restriction of the first series to coset representatives for $W / W_{J}$. We also show that the series has an explicit factorization involving the degrees of the free generators of the polynomial invariants of a canonically associated reflection group.
\end{abstract}

\section{INTRODUCTION}

1.1. Overview. Let $\Phi$ be a finite crystallographic root system with Weyl group $W$. In [3], Brenti and Carnevale consider the sign-twisted Poincaré series

$$
F(\Phi ; q):=\sum_{w \in W} \operatorname{sgn}(w) q^{\ell_{1}(w)},
$$

where $\ell_{1}(w)$ denotes the number of "odd inversions" of $w$; i.e. the number of positive roots of odd height mapped to negative roots by $w$. In particular, they give an explicit product formula for the above series in all cases except $E_{8}$. For the root systems $B_{n}$ and $C_{n}$, they also have multivariate refinements that keep track of more detailed information about the odd inversions, and in earlier work for types $A$ and $B$ [4], they consider variations that include restricting the series to the distinguished coset representatives for any parabolic quotient $W / W_{J}$.

Among their motivations, we should mention work of Klopsch and Voll [6]. In the course of enumerating flags of subspaces over a finite field that are non-degenerate with respect to various classes of bilinear forms, they conjectured a formula (proved in [4]) that amounted to an explicit evaluation of the above series restricted to any parabolic quotient in type $A$. A similar explicit evaluation for parabolic quotients in type $B$ arose conjecturally in the work of Stasinski and Voll [11] on the zeta functions for certain group schemes. (This conjecture was proved independently in [4] and [8].)

The main goal of the present article is to provide further insights into these signtwisted series, with an emphasis on unified, classification-free results. For example, it seems natural from the perspective of general root systems to exploit the fact that the reflections corresponding to the even roots permute the odd roots. Although this

Manuscript received 24th July 2018, revised 17th January 2019, accepted 21 st January 2019.

KEYWORds. Weyl group, root system, Poincaré series, inversion. 
diverges somewhat from the point of view taken in [3] (see Remark 6.2 below), it leads to multivariate refinements that share the common feature that odd roots in the same "even orbit" are given the same weight. Thus the multivariate series we consider here involve 2 variables for $A_{n}, B_{n}, F_{4}$ and $G_{2}$, and 4 variables for $C_{n}$.

Our main results are as follows:

1. A combinatorial quotient theorem proving that for every parabolic subsystem $\Phi_{J}$, the corresponding series $F\left(\Phi_{J} ; q\right)$ is a divisor of $F(\Phi ; q)$ (see Theorem 5.1 and its corollaries). This makes it clear a priori (i.e. without use of the classification of finite root systems) that the series $F(\Phi ; q)$ necessarily has many irreducible factors.

2. An explicit product formula for the multivariate series for each irreducible root system (Theorem 6.1). The proof is by induction with respect to rank, using Theorem 5.1. Each inductive step requires the evaluation of a sign-twisted sum over a small number of right coset representatives. In the classical cases, there are only 2 or 4 terms in each sum.

3. A general result that identifies, in a classification-free way, sufficient conditions for the multivariate series for a parabolic quotient $W / W_{J}$ to be the ratio of the corresponding series for $W$ and $W_{J}$ (Theorem 7.1). Conjecturally, the conditions are also necessary.

4. A uniform presentation of the univariate product formula, revealing that

$$
F(\Phi ; q)=\left(1-q^{a_{1}}\right) \cdots\left(1-q^{a_{k}}\right),
$$

where $a_{1}, \ldots, a_{k}$ are the degrees of the free generators of the polynomial invariants of a certain reflection group canonically associated to $\Phi$ (Theorem 8.5). Although our only proof of this formula is case-by-case, it does suggest a possible framework for a classification-free approach to the factorization of $F(\Phi ; q)$.

1.2. Preliminaries. Throughout, $V$ shall denote a real Euclidean space with inner product $\langle\cdot, \cdot\rangle$ and $\Phi \subset V$ a finite crystallographic root system with co-root system $\Phi^{\vee}=\left\{\beta^{\vee}: \beta \in \Phi\right\}$, positive roots $\Phi^{+}$, and Weyl group $W=W(\Phi)$. We use a common index set $I$ for the simple roots $\left\{\alpha_{i}: i \in I\right\} \subset \Phi$ and simple reflections $\left\{s_{i}: i \in I\right\} \subset W$. We will embed $\Phi^{\vee}$ in $V$ by taking $\beta^{\vee}=2 \beta /\langle\beta, \beta\rangle$.

If $L$ is a sublattice of the root lattice $\mathbb{Z} \Phi$, it is easy to show that $\Phi \cap L$ is again a root system in $V$, possibly of lower rank. The main lattice of interest here is

$$
L_{0}:=\left\{\sum a_{i} \alpha_{i}: a_{i} \in \mathbb{Z}, \sum a_{i} \text { even }\right\} .
$$

This has index 2 in $\mathbb{Z} \Phi$, and thus partitions $\Phi$ into "even" and "odd" roots; i.e.

$$
\Phi=\Phi_{0} \cup \Phi_{1},
$$

where $\Phi_{0}=\Phi \cap L_{0}$ is the root subsystem consisting of all roots of even height, and $\Phi_{1}$ is the set of roots with odd height. Note that the even Weyl group $W\left(\Phi_{0}\right)$ necessarily permutes $\Phi_{0}$ and $\Phi_{1}$.

Recall that the length function on $W$ counts inversions; i.e. $\ell(w)=|\Phi(w)|$, where

$$
\Phi(w)=\left\{\beta \in \Phi^{+}:-w \beta \in \Phi^{+}\right\} .
$$

We may thus refine length by separating the contributions of even and odd roots; i.e.

$$
\ell(w)=\ell_{0}(w)+\ell_{1}(w),
$$

where $\ell_{i}(w):=\left|\Phi_{i}(w)\right|$ and $\Phi_{i}(w):=\Phi_{i} \cap \Phi(w)$.

As discussed earlier, our central object of interest is the series

$$
F(\Phi ; q):=\sum_{w \in W} \operatorname{sgn}(w) q^{\ell_{1}(w)}
$$


Note that since every nontrivial $w \in W$ inverts at least one simple (and therefore odd) root, it follows that $F(\Phi ; q)$ has constant term 1 . Likewise, since there is a unique element that inverts all of the simple roots (the longest element), it is therefore also the unique element that inverts all of the odd roots. Thus the degree of $F(\Phi ; q)$ is the number of odd positive roots.

It would be reasonable to consider a generalization of $(2)$ in which the pair $\left(\Phi_{0}, \Phi_{1}\right)$ is replaced with $\left(\Phi^{\prime}, \Phi \backslash \Phi^{\prime}\right)$ for any root subsystem $\Phi^{\prime} \subset \Phi$. However, it should be noted that the corresponding series will vanish unless $\Phi^{\prime}$ contains none of the simple roots of $\Phi$. Indeed, the simple reflection $s_{i}$ has only one inversion: the simple root $\alpha_{i}$. If this simple root belongs to $\Phi^{\prime}$, then $s_{i}$ must necessarily permute $\Phi^{+} \backslash \Phi^{\prime}$. Thus multiplication by $s_{i}$ would change the sign while preserving the number of inversions not in $\Phi^{\prime}$. That is, the contributions from pairs $\left(w, w s_{i}\right)$ would cancel out.

We remark that each equal rank real form of a complex semisimple Lie group with root system $\Phi$ induces a parity function on $\Phi$ (more precisely, a homomorphism $\mathbb{Z} \Phi \rightarrow \mathbb{Z} / 2 \mathbb{Z}$ ) defined up to the action of $W$. The even roots in this setting correspond to the roots of the maximal compact subgroup. Thus the parity function we are concerned with here is associated to the quasisplit equal rank real form; i.e. the case where there is a positive system in which all simple roots have odd parity. (See for example [7], especially Chapter VI and the discussion surrounding (6.99).)

For each $J \subseteq I$, we let $\Phi_{J}$ denote the parabolic subsystem of $\Phi$ generated by $\left\{\alpha_{j}: j \in J\right\}$, and $W_{J}=W\left(\Phi_{J}\right)=\left\langle s_{j}: j \in J\right\rangle$ the associated Weyl group. It is well known that

$$
\begin{aligned}
& W^{J}:=\left\{w \in W: \ell\left(w s_{j}\right)>\ell(w) \text { for all } j \in J\right\} \\
& { }^{J} W:=\left\{w \in W: \ell\left(s_{j} w\right)>\ell(w) \text { for all } j \in J\right\}
\end{aligned}
$$

are the unique representatives of minimum length for the left and right cosets of $W_{J}$ in $W$, respectively. Moreover, one has $\ell(x y)=\ell(x)+\ell(y)$ for all $x \in W^{J}, y \in W_{J}$ and all $x \in W_{J}, y \in{ }^{J} W$.

The following key feature of inversion sets will be used repeatedly.

LEMma 1.1. For all $x, y \in W$ such that $\ell(x y)=\ell(x)+\ell(y)$, we have

$$
\Phi(x y)=\Phi(y) \dot{\cup} y^{-1} \Phi(x) \quad \text { (disjoint union). }
$$

Proof. It is clear that if $\beta \in \Phi^{+}$is an inversion of $x y$, then either $y \beta<0$, or $y \beta>0$ and $x y \beta<0$; i.e. $\beta \in \Phi(y)$ or $y \beta \in \Phi(x)$. Thus $\Phi(x y) \subseteq \Phi(y) \cup y^{-1} \Phi(x)$. If this union failed to be disjoint, or the inclusion was proper, then we would have

$$
\ell(x y)=|\Phi(x y)|<|\Phi(x)|+|\Phi(y)|=\ell(x)+\ell(y),
$$

a contradiction.

\section{ORBITS OF ODD ROOTS}

Recall that the even Weyl group $W\left(\Phi_{0}\right)$ permutes the odd roots; the structure of these orbits will play a key role in what follows. The $W$-orbit structure is well-known: two roots (whether or not they are odd) belong to the same $W$-orbit if and only if they belong to the same irreducible component of $\Phi$ and have the same length. Moreover, at most two root lengths (long and short) occur within any irreducible component.

LEMMA 2.1. Let $\gamma_{1}, \gamma_{2}$ be distinct odd roots such that $\left\langle\gamma_{2}, \gamma_{1}^{\vee}\right\rangle>0$.

(a) If $\gamma_{1}$ and $\gamma_{2}$ have the same length, then they belong to the same $W\left(\Phi_{0}\right)$-orbit.

(b) If $\gamma_{1}$ is long and $\gamma_{2}$ is short, then $\gamma_{1}=\gamma_{2}+\beta$, where $\beta \in \Phi_{0}$ and $\left\langle\beta, \gamma_{1}^{\vee}\right\rangle=1$. 
Proof. In either case, we must have $\left\langle\gamma_{2}, \gamma_{1}^{\vee}\right\rangle=1$ (e.g. see [1, VI.1.3]). Hence the reflection corresponding to $\gamma_{1}$ maps $\gamma_{2}$ to $-\beta$ where $\beta=\gamma_{1}-\gamma_{2}$ is a necessarily even root of the same length as $\gamma_{2}$. Furthermore, $\left\langle\beta, \gamma_{1}^{\vee}\right\rangle=\left\langle\gamma_{1}-\gamma_{2}, \gamma_{1}^{\vee}\right\rangle=1$, so the conclusions in (b) hold for either case. In (a), the root $\beta$ must also have the same length as $\gamma_{1}$, so $\left\langle\gamma_{1}, \beta^{\vee}\right\rangle=\left\langle\beta, \gamma_{1}^{\vee}\right\rangle=1$ and the (even) reflection corresponding to $\beta$ maps $\gamma_{1}$ to $\gamma_{1}-\beta=\gamma_{2}$.

LEMma 2.2. Let $\mathcal{O}_{1}, \mathcal{O}_{2}$ be $W\left(\Phi_{0}\right)$-orbits generated by odd roots of the same length. If $\mathcal{O}_{1}-\mathcal{O}_{2} \subset \operatorname{Span} \Phi_{0}$, then $\mathcal{O}_{1}= \pm \mathcal{O}_{2}$ or $\mathcal{O}_{1}, \mathcal{O}_{2} \subset \operatorname{Span} \Phi_{0}$.

Proof. Let $V_{0}=\operatorname{Span} \Phi_{0}$ and assume $\mathcal{O}_{1} \neq \pm \mathcal{O}_{2}$. If we select arbitrary representatives $\gamma_{i}$ from $\mathcal{O}_{i}$, then $\gamma_{1} \neq \pm \gamma_{2}$ and Lemma 2.1(a) (applied to both $\gamma_{2}$ and $-\gamma_{2}$ ) shows that $\gamma_{1}$ and $\gamma_{2}$ must be orthogonal. Hence $\operatorname{Span} \mathcal{O}_{1}$ and $\operatorname{Span} \mathcal{O}_{2}$ are orthogonal as well. On the other hand, the $W\left(\Phi_{0}\right)$-orbit generated by any vector $\gamma$ is spanned by $\gamma$ and the irreducible components of $\Phi_{0}$ for which the orthogonal projection of $\gamma$ is nonzero. In particular, the span of such an orbit contains the orthogonal projection of $\gamma$ onto $V_{0}$, and hence the projection onto $V_{0}^{\perp}$ as well. However, we are given that $\gamma_{1}-\gamma_{2} \in V_{0}$, so $\gamma_{1}$ and $\gamma_{2}$ have the same orthogonal projection onto $V_{0}^{\perp}$. Thus the projections cannot be orthogonal unless they are 0 ; i.e. $\gamma_{1}, \gamma_{2} \in V_{0}$.

Lemma 2.3. If $\Phi$ and $\Phi_{0}$ have the same rank and $\Phi$ is irreducible, then any two odd roots of the same length are in the same $W\left(\Phi_{0}\right)$-orbit.

Proof. Since $\Phi$ and $\Phi_{0}$ are assumed to have the same rank, every root in $\Phi$ must be in the linear span of $\Phi_{0}$. Thus given any odd root $\gamma$ that is $\Phi_{0}$-dominant, we may consider its coordinates with respect to the simple roots of $\Phi_{0}$, say $\gamma=\sum c_{i} \beta_{i}$. One knows that dominance forces $c_{i} \geqslant 0$ and the support (i.e. $\left\{i: c_{i}>0\right\}$ ) must coincide with the union of one or more irreducible components of $\Phi_{0}$. (This amounts to the fact that the inverse of any irreducible Cartan matrix has strictly positive entries.)

Now suppose that $\gamma_{1}, \gamma_{2}, \ldots$ are the $\Phi_{0}$-dominant members of the distinct $W\left(\Phi_{0}\right)$ orbits of odd roots. If $\gamma_{1}$ and $\gamma_{2}$ have the same length and overlapping support, the dominance forces $\left\langle\gamma_{2}, \gamma_{1}^{\vee}\right\rangle>0$ and we contradict Lemma 2.1(a). In other words, distinct orbits generated by odd roots of the same length have disjoint, orthogonal support.

Alternatively, suppose that $\gamma_{1}$ is long and $\gamma_{2}$ is short. If they have overlapping support, we still have $\left\langle\gamma_{2}, \gamma_{1}^{\vee}\right\rangle>0$ and Lemma 2.1(b) implies that $\gamma_{1}=\gamma_{2}+\beta$ where $\beta$ is an even root such that $\left\langle\beta, \gamma_{1}^{\vee}\right\rangle=1$. The latter forces $\beta$ to be positive, since $\gamma_{1}$ is dominant. Thus the support of $\gamma_{1}$ contains the support of $\gamma_{2}$. However, an even root such as $\beta$ must have support that is confined to a single irreducible component of $\Phi_{0}$, so if there is a gap between the supports of $\gamma_{1}$ and $\gamma_{2}$, it is this one irreducible component, and $\beta$ is the orthogonal projection of $\gamma_{1}$ onto this component.

Returning to the hypothesis that $\gamma_{1}$ and $\gamma_{2}$ have the same length, consider that our task is to show that this leads to a contradiction. Since $\gamma_{1}$ and $\gamma_{2}$ must have disjoint orthogonal support and $\Phi$ is irreducible, this is possible only if another orbit representative, say $\gamma_{3}$, has support overlapping $\gamma_{1}$ or $\gamma_{2}$ or both. As we have seen, this is possible only if $\gamma_{3}$ is long and $\gamma_{1}$ and $\gamma_{2}$ are short. Since this eliminates the possibility of $\gamma_{1}$ and $\gamma_{2}$ both being long, this means that $\gamma_{3}$ generates the only long odd orbit, and irreducibility forces its support to include the supports of both $\gamma_{1}$ and $\gamma_{2}$. Furthermore, since the supports of $\gamma_{1}$ and $\gamma_{2}$ are disjoint, the inclusions must be proper, and as we have shown, each "support gap" must be a single irreducible component of $\Phi_{0}$. This forces the support of $\gamma_{3}$ to consist of exactly two irreducible components, and the orthogonal projections of $\gamma_{3}$ onto these two components must be even roots, contradicting the fact that $\gamma_{3}$ is odd. 


\section{Proposition 2.4.}

(a) Every $W\left(\Phi_{0}\right)$-orbit of odd roots includes a simple or a negative simple root.

(b) If the nodes of the Dynkin diagram of $\Phi$ are 2-colored so that each edge is incident to a black vertex and a white vertex, then the black simple roots and the negatives of the white simple roots in the same $W$-orbit belong to the same $W\left(\Phi_{0}\right)$-orbit.

(c) If $\Phi$ is irreducible of rank $n$, then either

(i) $\Phi_{0}$ has rank $n-1$, all simple roots of $\Phi_{0}$ have height 2 in $\Phi$, and every opposite pair of odd roots $\pm \gamma$ belong to distinct $W\left(\Phi_{0}\right)$-orbits, or

(ii) $\Phi_{0}$ has rank $n$, one simple root of $\Phi_{0}$ has height 4 in $\Phi$, and all odd roots of the same length are in the same $W\left(\Phi_{0}\right)$-orbit.

Furthermore, case (i) occurs if and only if $\Phi \cong A_{n}$ or $C_{n}$.

Proof. Choose a black-white coloring of the Dynkin diagram as in (b), and assume that $\Phi$ is irreducible of rank $n$. Since the diagram is a tree, there are exactly $n-1$ roots $\beta_{1}, \ldots, \beta_{n-1}$ of height 2 in $\Phi$; each is of the form $\alpha_{i}+\alpha_{j}$ where $i$ is a black node and $j$ is an adjacent white node. If the edge between $i$ and $j$ is simple (i.e. the parabolic subsystem generated by $\alpha_{i}$ and $\alpha_{j}$ is isomorphic to $A_{2}$ ), then the reflection corresponding to $\alpha_{i}+\alpha_{j}$ interchanges $\alpha_{i}$ with $-\alpha_{j}$ and $-\alpha_{i}$ with $\alpha_{j}$. It follows that for any nodes $i$ and $j$ connected by a path of simple edges, the $W\left(\Phi_{0}\right)$-orbit of $\alpha_{i}$ contains $\alpha_{j}$ if $i$ and $j$ have the same color, and the orbit contains $-\alpha_{j}$ if $i$ and $j$ have different colors. On the other hand, it is well-known that two simple (or negative simple) roots belong to the same $W$-orbit if and only if they are in the same connected component of the Dynkin diagram after all non-simple edges have been deleted. Thus (b) follows.

Now consider that as a member of the even root system $\Phi_{0}$, each root $\beta_{i}$ is clearly indecomposable (i.e. not expressible as a sum of two positive even roots), and thus is necessarily a simple root of $\Phi_{0}$. On the other hand, as a root subsystem of a rank $n$ root system, $\Phi_{0}$ necessarily has rank $\leqslant n$, and thus it can have at most one additional indecomposable root.

CASE 1: $\Phi_{0}$ HAS RANK $n-1$. Define an integral co-weight $\omega$ for $\Phi$ by setting $\left\langle\alpha_{i}, \omega\right\rangle=1$ (if $i$ is black) and -1 (if $i$ is white). We therefore have $\left\langle\beta_{i}, \omega\right\rangle=0$ for all $i$ and hence $W\left(\Phi_{0}\right)$ fixes $\omega$, since $\beta_{1}, \ldots, \beta_{n-1}$ forms a base for $\Phi_{0}$ in this case. Thus $\langle\gamma, \omega\rangle$ is a constant as $\gamma$ varies over any $W\left(\Phi_{0}\right)$-orbit in $\Phi$. For odd $\gamma$, this constant is the sum of an odd number of \pm 1 's, and hence nonzero. Consequently, the $W\left(\Phi_{0}\right)$-orbits of $\gamma$ and $-\gamma$ are distinct for every odd root $\gamma$, proving (i).

We claim that $\langle\gamma, \omega\rangle$ is limited to the values $0, \pm 1$ for all $\gamma \in \Phi$ (i.e. $\omega$ is minuscule). Indeed, even roots belong to the hyperplane orthogonal to $\omega$, and if there were an odd root $\gamma$ such that $\langle\gamma, \omega\rangle \geqslant 3$, then there would be an even root of the form $\beta=\gamma \pm \alpha_{i}$ for some $i$. In that case, we would have $\langle\beta, \omega\rangle \geqslant 2$, a contradiction.

Thus every $W\left(\Phi_{0}\right)$-orbit of odd roots belongs to the (affine) hyperplane $\langle\cdot, \omega\rangle=1$ or its negative, and both hyperplanes contain at least one simple root or negative simple root from each $W$-orbit. To prove (a) for this case, it therefore suffices to show that all roots of a given length within one of these hyperplanes belong to the same $W\left(\Phi_{0}\right)$-orbit. If there were two such orbits, say $\mathcal{O}_{1}$ and $\mathcal{O}_{2}$, then we cannot have $\mathcal{O}_{1}= \pm \mathcal{O}_{2}$ (the orbit opposite to $\mathcal{O}_{1}$ is in the opposite hyperplane), and yet we do have $\left\langle\gamma_{1}-\gamma_{2}, \omega\right\rangle=0$ for all $\gamma_{i} \in \mathcal{O}_{i}$, so Lemma 2.2 implies $\mathcal{O}_{i} \subset \operatorname{Span} \Phi_{0}$, a contradiction.

To see that $\Phi=A_{n}$ and $C_{n}$ belong to Case 1, it suffices to show that the even roots are confined to a central hyperplane in these cases. For this, let $\varepsilon_{1}, \ldots, \varepsilon_{n}$ be the standard orthornomal basis for $\mathbb{R}^{n}$, and realize $C_{n}$ using the simple roots $\alpha_{1}=2 \varepsilon_{1}$ and $\alpha_{i}=\varepsilon_{i}-\varepsilon_{i-1}(1<i \leqslant n)$. It is easy to see that $\omega=(1 / 2)(1,-1,1,-1, \ldots) \in \mathbb{R}^{n}$ 
has the property that $\left\langle\alpha_{i}, \omega\right\rangle= \pm 1$ for all $i$, so $\beta$ is even if and only if $\langle\beta, \omega\rangle$ is even. On the other hand, the value $\langle\beta, \omega\rangle$ for any $\operatorname{root} \beta$ is the sum or difference of two coordinates of $\omega$, so the only possible values are 0 and \pm 1 . That is, $\beta$ is even if and only if $\langle\beta, \omega\rangle=0$ and thus $\Phi_{0}$ is confined to the hyperplane orthogonal to $\omega$. This yields the same result for $A_{n-1}$ if we recognize it as a parabolic subsystem of $C_{n}$. That is, the even roots of $A_{n-1}$ are confined to the hyperplane orthogonal to $\omega$ in the span of $A_{n-1}$.

CASE 2: $\Phi_{0}$ HAS RANK $n$. In this case, there must be a positive root $\beta_{0} \in \Phi_{0}$ that has height at least 4 in $\Phi$ and is indecomposable in $\Phi_{0}$. Any such root is necessarily a simple root of $\Phi_{0}$ and therefore unique, since the height 2 roots already account for all but one of the simple roots of $\Phi_{0}$. Having previously shown in Lemma 2.3 that length separates the $W\left(\Phi_{0}\right)$-orbits of odd roots in this case, (a) follows immediately. To complete the proof, it suffices to identify an indecomposable even root of height 4 in all remaining irreducible $\Phi$ (i.e. other than $A_{n}$ or $C_{n}$ ). For this, note that all such $\Phi$ must have a parabolic subsystem isomorphic to $D_{4}, B_{3}$, or $G_{2}$.

Case 2(a) $\Phi$ includes a parabolic copy of $D_{4}$. Here, we claim that the sum of the four simple roots in the $D_{4}$-subsystem is an indecomposable root $\beta_{0}$ of $\Phi_{0}$. If not, this root would have to be a sum of two height 2 roots, and hence the diagram of $D_{4}$ would have to have two white vertices and two black vertices.

Case 2(b) $\Phi$ includes a parabolic copy of $B_{3}$. Numbering the Dynkin diagram of this subsystem $1 \Leftarrow 2-3$, we claim that $\beta_{0}=2 \alpha_{1}+\alpha_{2}+\alpha_{3}$ is indecomposable in $\Phi_{0}$. This is immediate after confirming that $\beta_{0}$ is indeed a root, since it is a sum of unequal numbers of white and black simple roots (counted with multiplicity).

Case 2(c) $\Phi$ is isomorphic to $G_{2}$. With the simple roots indexed so that $\alpha_{1}$ is short and $\alpha_{2}$ is long, the root $\beta_{0}=3 \alpha_{1}+\alpha_{2}$ is clearly indecomposable in $\Phi_{0}$.

\begin{tabular}{|c|c|c|c|c|c|c|c|c|c|c|c|}
\hline$\Phi$ & $A_{2 n-1}$ & $A_{2 n}$ & $B_{n}$ & $C_{n}$ & $D_{2 n-1}$ & $D_{2 n}$ & $E_{6}$ & $E_{7}$ & $E_{8}$ & $F_{4}$ & $G_{2}$ \\
\hline$\left|\Gamma\left(\Phi_{0}\right)\right|$ & 2 & 1 & 2 & 2 & 2 & 4 & 1 & 2 & 1 & 1 & 1 \\
$\left|W\left(\Phi_{0}\right) \backslash \Phi_{1}\right|$ & 2 & 2 & 2 & 4 & 1 & 1 & 1 & 1 & 1 & 2 & 2 \\
\hline
\end{tabular}

TABLE 1 . The order of $\Gamma\left(\Phi_{0}\right)$ and the $W\left(\Phi_{0}\right)$-orbit count on $\Phi_{1}$.

\section{REMARKS 2.5 .}

(a) The above result implies that a $W\left(\Phi_{0}\right)$-orbit of odd roots may fail to include a simple root only if $\Phi$ is of type $A$ or $C$ and the corresponding $W$-orbit includes only one simple root. That is, only for one of the long odd orbits in $C_{n}$, a long and a short orbit in $C_{2}$, and one of the odd orbits in $A_{1}$. Most of these orbits do include positive roots, the exceptions being the singleton orbits formed by the short negative simple root of $C_{2}$, and the negative simple root of $A_{1}$.

(b) To minimize the need for special considerations for the root system $B_{2} \cong C_{2}$, it is preferable to require the type $B$ series of root systems to begin at rank 3 and allow rank 2 only for type $C$. That way, all type $B$ root systems have only 2 odd orbits, whereas in all type $C$ cases there are 4 . See the last row of Table 1. 


\section{The EVEN NORMALIZER}

It will develop that the series $F(\Phi ; q)$ depends only on a subgroup $N\left(\Phi_{0}\right)$ of $W$; namely, the normalizer of $W\left(\Phi_{0}\right)$ in $W$. Since the $W$-action on $\Phi$ corresponds to the conjugation action of $W$ on reflections, we have that

$$
N\left(\Phi_{0}\right)=\left\{w \in W: w \Phi_{0}=\Phi_{0}\right\} .
$$

Let $\Delta_{0}$ denote the base formed by the simple roots for $\Phi_{0}$. Since the action of $W\left(\Phi_{0}\right)$ on bases for $\Phi_{0}$ is simply transitive, the normalizer is a semidirect product; i.e.

$$
N\left(\Phi_{0}\right) \cong W\left(\Phi_{0}\right) \rtimes \Gamma\left(\Phi_{0}\right),
$$

where $\Gamma\left(\Phi_{0}\right):=\left\{w \in W: w \Delta_{0}=\Delta_{0}\right\}$ is the subgroup of $N\left(\Phi_{0}\right)$ consisting of elements with no even inversions. See Table 1 for the cardinalities of $\Gamma\left(\Phi_{0}\right)$ for irreducible $\Phi$.

The normalizers $N\left(\Phi_{0}\right)$ are closely related to the groups of "chessboard" elements for classical Weyl groups discussed in [4], [3], [6], and [10]. In fact, for the root systems $A_{n}, B_{2 n+1}$, and $D_{n}$ they are exactly the same, whereas for $B_{2 n}$, the chessboard group defined in [4] and [10] may be seen as the normalizer of a reflection subgroup of $W$ that is slightly larger than $W\left(\Phi_{0}\right)$.

To illustrate the case $\Phi=B_{2 n}(n \geqslant 2)$ in more detail, fix a choice of simple roots $\alpha_{1}=\varepsilon_{1}$ and $\alpha_{i}=\varepsilon_{i}-\varepsilon_{i-1}(1<i \leqslant 2 n)$. From the construction in the proof of Proposition 2.4, one obtains that the simple even roots are $\varepsilon_{2}, \varepsilon_{3}+\varepsilon_{1}$, and $\varepsilon_{i}-\varepsilon_{i-2}$ $(2<i \leqslant 2 n)$, so $\Phi_{0}$ decomposes orthogonally into $\left\{ \pm \varepsilon_{2 i}, \pm \varepsilon_{2 i} \pm \varepsilon_{2 j}: i \neq j\right\} \cong B_{n}$ and $\left\{ \pm \varepsilon_{2 i+1} \pm \varepsilon_{2 j+1}: i \neq j\right\} \cong D_{n}$. Recognizing that $W$ acts via signed permutations of the coordinates, one sees that $N\left(\Phi_{0}\right) \cong W\left(B_{n}\right) \times W\left(B_{n}\right)$, with the subgroup $\Gamma\left(\Phi_{0}\right)$ being a 2-element group generated by the (simple) reflection corresponding to $\varepsilon_{1}$. Thus it happens in this case that $N\left(\Phi_{0}\right)$ is also a reflection subgroup of $W$, and the chessboard group cited above may be seen as its $W$-normalizer.

\section{REMARKS 3.1.}

(a) The longest element $w_{0}=w_{0}(\Phi)$ in $W(\Phi)$ maps roots of height $h$ to height $-h$, so it preserves parity (i.e. belongs to $N\left(\Phi_{0}\right)$ ) and maps the simple roots of $\Phi_{0}$ to some permutation of their negatives. Since $w_{0}\left(\Phi_{0}\right)$ has a similar effect on the even roots, it follows that $w_{0}\left(\Phi_{0}\right) w_{0}(\Phi)$ is always a member of $\Gamma\left(\Phi_{0}\right)$ (possibly trivial).

(b) It is tempting to assume that $\Gamma=\Gamma\left(\Phi_{0}\right)$ acts faithfully as a group of diagram automorphisms of $\Phi_{0}$, but this isn't true in general: a nontrivial $w \in \Gamma$ may act trivially on $\Delta_{0}$ and $\Phi_{0}$. For example, if $\Phi$ is $C_{2}$, then $\Phi_{0}$ has rank 1 and therefore has no nontrivial diagram automorphisms. However, $\Gamma$ is a 2 -element group in this case.

Since $\Gamma\left(\Phi_{0}\right) \cong N\left(\Phi_{0}\right) / W\left(\Phi_{0}\right)$, it follows that $\Gamma\left(\Phi_{0}\right)$ acts on the $W\left(\Phi_{0}\right)$-orbits of odd roots (and generally, the $W\left(\Phi_{0}\right)$-orbits of any $N\left(\Phi_{0}\right)$-action). It will be important in what follows to identify the cases where the action on these orbits is nontrivial.

\section{Proposition 3.2.}

(a) If $\Phi$ is irreducible, then $\Gamma\left(\Phi_{0}\right)$ stabilizes each $W\left(\Phi_{0}\right)$-orbit of odd roots except possibly when $\Phi \cong A_{n}$ or $C_{n}$.

(b) If $\Phi \cong A_{2 n}$, then $\Gamma\left(\Phi_{0}\right)$ is trivial.

(c) If $\Phi \cong A_{2 n-1}$ or $C_{n}$, then $\left|\Gamma\left(\Phi_{0}\right)\right|=2$.

Moreover, in (c), if the diagram is properly 2-colored so that the black nodes include all end nodes (for $A_{2 n-1}$ ), or the end node corresponding to a short simple root (for $\left.C_{n}\right)$, then the nontrivial element of $\Gamma\left(\Phi_{0}\right)$ is the product of the black simple reflections, and it acts by interchanging opposite pairs $\pm \mathcal{O}$ of $W\left(\Phi_{0}\right)$-orbits of odd roots. 
Proof. By Proposition 2.4(c), we know that unless $\Phi$ is of type $A$ or $C$, odd roots in the same $W$-orbit are also in the same $W\left(\Phi_{0}\right)$-orbit. This proves (a).

For the remaining assertions, we may assume that $\Phi \cong A_{n}$ or $C_{n}$. In such cases, recall from the proof of Proposition 2.4 that there is an integral co-weight $\omega$ such that $\left\langle\alpha_{i}, \omega\right\rangle=1$ if $i$ is black and $\left\langle\alpha_{i}, \omega\right\rangle=-1$ if $i$ is white. (For $A_{2 n}$, any proper 2-coloring will suffice for what follows.) Moreover, the span of $\Phi_{0}$ is the hyperplane $V_{0}$ orthogonal to $\omega$, and hence $W\left(\Phi_{0}\right)$ is the stabilizer of $\omega$.

On the other hand, $N\left(\Phi_{0}\right)$ is the stabilizer of $V_{0}$. As a group of orthogonal transformations, it must therefore preserve the orthogonal complement of $V_{0}$. Hence

$$
N\left(\Phi_{0}\right)=\{x \in W: x \omega= \pm \omega\} .
$$

It follows that $\left|\Gamma\left(\Phi_{0}\right)\right| \leqslant 2$, with equality if and only if there is an element $x \in W$ such that $x \omega=-\omega$. In that case, $x$ acts on $W\left(\Phi_{0}\right)$-orbits by interchanging opposite pairs.

In the case $\Phi \cong A_{2 n}$, if we embed $\Phi$ in $\mathbb{R}^{2 n+1}$ so that $W$ acts by permuting coordinates, then $\omega$ will have $n+1$ equal positive coordinates and $n$ equal negative coordinates (or vice-versa), and thus no member of $W$ can transform $\omega$ to $-\omega$.

For the remaining cases, let $\theta=\sum \alpha_{i}^{\vee}$, where $i$ ranges over the black nodes. We claim that $\omega=\theta / 2$. To see this, consider that if $i$ is black, then none of its neighbors participate in the sum and $\left\langle\alpha_{i}, \theta\right\rangle=2$. If $j$ is white, then in most cases it has two neighbors, both black, with corresponding simple roots $\alpha_{i}$ that are not shorter than $\alpha_{j}$. Hence $\left\langle\alpha_{j}, \alpha_{i}^{\vee}\right\rangle=-1$ for both neighbors and $\left\langle\alpha_{j}, \theta\right\rangle=-2$. The one exception occurs in $C_{2 n}$ when $\alpha_{j}$ is long, in which case it has only one neighbor $i$, but $\left\langle\alpha_{j}, \alpha_{i}^{\vee}\right\rangle=-2$ and again $\left\langle\alpha_{j}, \theta\right\rangle=-2$. Either way, the claim follows.

Now let $x$ be the product of the black simple reflections. Since these reflections commute, it is clear that $\Phi(x)$ consists of the black simple roots, and it is evident from the co-root coordinates for $\theta$ that $x \omega=-\omega$. Hence $x$ belongs to the nontrivial coset of $N\left(\Phi_{0}\right)$. Since it has no even inversions, it is therefore a member of $\Gamma\left(\Phi_{0}\right)$.

\section{Parabolic and multivariate Refinements}

Let $\mathbf{q}=\left(q_{\beta}: \beta \in \Phi_{1}^{+}\right)$be a collection of variables indexed by the odd positive roots and consider the series

$$
F(\Phi ; \mathbf{q}):=\sum_{w \in W} \operatorname{sgn}(w) \prod_{\beta \in \Phi_{1}(w)} q_{\beta} .
$$

Of course we recover the original series $F(\Phi ; q)$ by setting $q_{\beta}=q$ for all $\beta \in \Phi_{1}^{+}$. More generally, for each $J \subseteq I$, we will consider the restriction of the above series to the set $W^{J}$ consisting of the minimum-length coset representatives for $W / W_{J}$; i.e.

$$
F_{J}(\Phi ; \mathbf{q}):=\sum_{w \in W^{J}} \operatorname{sgn}(w) \prod_{\beta \in \Phi_{1}(w)} q_{\beta} .
$$

One sees that $F_{J}(\Phi ; \mathbf{q})=F(\Phi ; \mathbf{q})$ when $J=\varnothing$

Although these multivariate series do not seem to offer simple closed forms, the following result shows that a significant amount of cancellation of terms does occur at this level of generality. (One should also note that this feature is specific to sums over left cosets. The analogous sum over ${ }^{J} W$ offers no comparable degree of cancellation, and specializations of such sums appear to factor less often than the left coset series. See Remark 7.6.)

Proposition 4.1. For all $J \subseteq I$, we have

$$
F_{J}(\Phi ; \mathbf{q})=\sum_{w \in N\left(\Phi_{0}\right) \cap W^{J}} \operatorname{sgn}(w) \prod_{\beta \in \Phi_{1}(w)} q_{\beta} .
$$


This justifies our prior claim that $F(\Phi ; q)$ depends only on the normalizer $N\left(\Phi_{0}\right)$.

For the proof, we need to fix a total ordering of the index set $I$. Given $w \in W$ such that $w \notin N\left(\Phi_{0}\right)$, we define the first fault of $w$ to be the least $i \in I$ such that $w^{-1} \alpha_{i}$ is even. Such an index $i$ must exist, otherwise, $w^{-1}$ would map all simple roots to odd roots, and thus $w^{-1}$ and $w$ would be parity preserving on roots; i.e. members of $N\left(\Phi_{0}\right)$.

LEMMA 4.2. If $w \in W^{J}$ and $w^{-1} \alpha_{i}$ is even for some $i \in I$, then $s_{i} w \in W^{J}$. Furthermore, if $i$ is the first fault of $w$, then $i$ is also the first fault of $s_{i} w$.

Proof. If $s_{i} w \notin W^{J}$, then there is some index $j \in J$ such that $\ell\left(s_{i} w s_{j}\right)<\ell\left(s_{i} w\right)$. Thus either $\ell(w)=\ell\left(s_{i} w s_{j}\right)$ or $\ell(w)=\ell\left(s_{i} w s_{j}\right)+2$. In the latter case, $w$ has a reduced expression that ends with $s_{j}$, contradicting the fact that $w \in W^{J}$. In the former case, the Deletion Property $[5, \S 1.7]$ implies that a reduced expression for $s_{i} w s_{j}$ may be obtained by taking a reduced expression for $w$, appending $s_{j}$, prepending $s_{i}$, and deleting two terms. If one or both of the deleted terms is internal to the chosen expression for $w$, then $w$ would have a reduced expression that begins with $s_{i}$ or ends with $s_{j}$. The latter contradicts the fact that $w \in W^{J}$, and the former contradicts the fact that $\ell\left(s_{i} w\right)>\ell(w)$ in this case. The remaining possibility is that the first and last terms are deleted; i.e. $s_{i} w s_{j}=w$, or equivalently, $w^{-1} \alpha_{i}= \pm \alpha_{j}$. However, this contradicts the fact that $w^{-1} \alpha_{i}$ is even.

For the second claim, consider that for all indices $k \in I$, we have

$$
\left(s_{i} w\right)^{-1} \alpha_{k}=w^{-1} s_{i} \alpha_{k}=w^{-1} \alpha_{k}-a w^{-1} \alpha_{i}
$$

where $a$ is the integer $\left\langle\alpha_{k}, \alpha_{i}^{\vee}\right\rangle$. Thus if $i$ is the first fault of $w$, it follows that $\left(s_{i} w\right)^{-1} \alpha_{k}$ is even if and only if $w^{-1} \alpha_{k}$ is even. In particular, $i$ must be the first fault of $s_{i} w$.

Proof of Proposition 4.1. Lemma 4.2 implies that the map $w \mapsto s_{i} w$ (with $i$ being the first fault of $w$ ) defines a sign-reversing involution on the members of $W^{J}$ not in $N\left(\Phi_{0}\right)$. Exchanging $w$ with $s_{i} w$ if necessary, we may assume that $\ell\left(s_{i} w\right)>\ell(w)$. In that case,

$$
\Phi\left(s_{i} w\right)=\Phi(w) \dot{\cup}\left\{w^{-1} \alpha_{i}\right\} .
$$

Thus the inversion sets of $w$ and $s_{i} w$ are identical aside from the presence of a single even root, so their contributions to the sum cancel out, leaving only the contributions from $N\left(\Phi_{0}\right) \cap W^{J}$.

REMARK 4.3. We have previously noted that the longest element $w_{0}$ is the unique member of $W$ that inverts all odd roots. Since $\Phi(w) \subseteq \Phi^{+} \backslash \Phi_{J}$ for all $w \in W^{J}$, we thus have the degree bound

$$
\operatorname{deg} F_{J}(\Phi ; q) \leqslant\left|\Phi_{1}^{+} \backslash \Phi_{J}\right|=\operatorname{deg} F(\Phi ; q)-\operatorname{deg} F\left(\Phi_{J} ; q\right) .
$$

Furthermore, there may be multiple members of $W^{J}$ that invert all of $\Phi_{1}^{+} \backslash \Phi_{J}$, resulting in the possibility of cancellation and a degree that does not achieve this bound. For example, when $\left(\Phi, \Phi_{J}\right) \cong\left(D_{4}, A_{1}^{\oplus 3}\right)$, there are 5 odd positive roots not in $\Phi_{J}$, but it is easily checked that $F_{J}\left(D_{4} ; q\right)=\left(1-q^{2}\right)\left(1+3 q^{2}\right)$. This also shows that the irreducible factors of $F_{J}(\Phi ; q)$ need not be cyclotomic.

From now on, we will focus on three specializations of the variables $q_{\beta}$; namely,

(i) The univariate grading; i.e. $q_{\beta}$ is independent of $\beta$.

(ii) The coarse grading; i.e. $q_{\beta}$ depends only on the $W$-orbit of $\beta$.

(iii) The fine grading; i.e. $q_{\beta}$ depends only on the $W\left(\Phi_{0}\right)$-orbit of $\beta$.

By Proposition 2.4, we know that in most irreducible cases, each $W$-orbit of roots contains only one $W\left(\Phi_{0}\right)$-orbit of odd roots, and hence, the coarse and fine gradings coincide. The exceptions are $A_{n}$ (which has two $W\left(\Phi_{0}\right)$-orbits of odd roots) and 
$C_{n}$ (which has four; two long and two short). Among these exceptions, $A_{1}$ and $C_{2}$ are somewhat degenerate in having an odd $W\left(\Phi_{0}\right)$-orbit that consists only of negative roots. (Recall Remark 2.5(a).) The corresponding series $F(\Phi ; \mathbf{q})$ under the fine grading therefore depends on only 1 or 3 variables, rather than 2 or 4 , respectively.

\section{Perfect cosets and divisibility}

Fix $J \subseteq I$ and consider a minimal right coset representative $y \in{ }^{J} W$. Recall that this means $\ell\left(s_{j} y\right)>\ell(y)$ for all $j \in J$, or equivalently $y^{-1} \alpha_{j}>0$ for such $j$. We say that $y$ is perfect if, in addition, $y^{-1} \alpha_{j}$ is odd for all $j \in J$. (Thus in the terminology of the previous section, the perfect members of ${ }^{J} W$ have no faults in $J$.) We let

$$
{ }^{J} W_{1}:=\left\{y \in W: y^{-1} \alpha_{j} \in \Phi_{1}^{+} \text {for all } j \in J\right\}
$$

denote the set of minimal coset representatives that are perfect, and

$$
G_{J}(\Phi ; \mathbf{q}):=\sum_{y \in \in^{J} W_{1}} \operatorname{sgn}(y) \prod_{\beta \in \Phi_{1}(y)} q_{\beta}
$$

the corresponding sign-twisted series.

THEOREM 5.1. For all $J \subseteq I$, we have

$$
F(\Phi ; \mathbf{q})=\sum_{y \in \in^{J} W_{1}}\left(\operatorname{sgn}(y) \prod_{\beta \in \Phi_{1}(y)} q_{\beta}\right) F\left(\Phi_{J} ; y^{-1} \mathbf{q}\right)
$$

under the fine $e^{(1)}$ grading for $\Phi$, where $y^{-1} \mathbf{q}$ denotes the change of variable $q_{\beta} \rightarrow q_{y^{-1} \beta}$ for odd $\beta \in \Phi_{J}^{+}$. In particular, if $F\left(\Phi_{J} ; y^{-1} \mathbf{q}\right)=F\left(\Phi_{J} ; \mathbf{q}\right)$ for all $y \in{ }^{J} W_{1}$, then

$$
F(\Phi ; \mathbf{q})=G_{J}(\Phi ; \mathbf{q}) F\left(\Phi_{J} ; \mathbf{q}\right)
$$

under the fine grading for $\Phi$.

Note that if $y$ is perfect and $\beta \in \Phi_{J}$ is odd, then $y^{-1} \beta$ is clearly another odd root in the same $W$-orbit. Hence, for the coarse specialization of the variables $q_{\beta}$, we have

Corollary 5.2. In all cases, $F(\Phi ; \mathbf{q})=G_{J}(\Phi ; \mathbf{q}) F\left(\Phi_{J} ; \mathbf{q}\right)$ under the coarse grading.

Thus it is clear a priori (i.e. without use of the classification of finite root systems, or explicit evaluation) that $F(\Phi ; \mathbf{q})$ has many divisors under the coarse grading, and therefore is likely to have a nice factorization.

For the finer gradings available with $A_{n}$ and $C_{n}$, it need not be the case that $F(\Phi ; \mathbf{q})$ is divisible by $F\left(\Phi_{J} ; \mathbf{q}\right)$. For example, a posteriori (see Theorem 6.1 below) $F\left(A_{n} ; \mathbf{q}\right)$ is divisible by $F\left(A_{k} ; \mathbf{q}\right)$ under the fine grading only if $k$ is even, or $n$ is odd and $k+1$ divides $n+1$. On the other hand, the following result shows that $A_{n}$ and $C_{n}$ have parabolic subsystems of co-rank at most 2 where divisibility is clear a priori.

Corollary 5.3. If $\Phi_{J} \cong A_{2 n}$, then $F(\Phi ; \mathbf{q})=G_{J}(\Phi ; \mathbf{q}) F\left(\Phi_{J} ; \mathbf{q}\right)$ in the fine grading.

Proof. For perfect $y \in{ }^{J} W$, the map $\beta \mapsto y^{-1} \beta$ acts as a parity-preserving isomorphism between two copies of $A_{2 n}$ embedded in $\Phi$ (namely, $\Phi_{J}$ and $y^{-1} \Phi_{J}$ ). Although it is possible that $\beta$ and $y^{-1} \beta$ are in distinct $W\left(\Phi_{0}\right)$-orbits for odd $\beta \in \Phi_{J}$, there is a diagram automorphism of $A_{2 n}$ that interchanges the black and white vertices of the 2-colored Dynkin diagram. By Proposition 2.4, it follows that this automorphism interchanges the two orbits of odd roots, and thus $F\left(A_{2 n} ; \mathbf{q}\right)$ is invariant under interchanging the variables $q_{\beta}$ in these two orbits. Thus either way, we have $F\left(\Phi_{J} ; y^{-1} \mathbf{q}\right)=F\left(\Phi_{J} ; \mathbf{q}\right)$ for all $y$.

\footnotetext{
${ }^{(1)}$ Note that the fine grading for $\Phi$ may force the identification of some variables appearing in $F\left(\Phi_{J} ; \mathbf{q}\right)$ that would otherwise be distinct in the fine grading for $\Phi_{J}$.
} 
Proof of Theorem 5.1. Suppose that $y \in{ }^{J} W$ is not perfect, so that there is some $j \in$ $J$ such that $\beta=y^{-1} \alpha_{j}$ is an even (necessarily positive) root. Since $\ell(x y)=\ell(x)+\ell(y)$ for all $x \in W_{J}$, Lemma 1.1 implies

$$
\Phi(x y)=\Phi(y) \dot{\cup} y^{-1} \Phi(x) .
$$

Replacing $x$ with $x s_{j}$ if necessary so that $\ell\left(x s_{j}\right)>\ell(x)$, we also have

$$
\Phi\left(x s_{j} y\right)=\Phi(y) \dot{\cup}\left\{y^{-1} \alpha_{j}\right\} \dot{\cup} y^{-1} s_{j} \Phi(x)=\Phi(y) \dot{\cup}\{\beta\} \dot{\cup} t y^{-1} \Phi(x),
$$

where $t=y^{-1} s_{j} y$ is the reflection corresponding to $\beta$. Since $\beta$ is even, $t$ belongs to $W\left(\Phi_{0}\right)$, so for each $W\left(\Phi_{0}\right)$-orbit of odd roots, the number of such roots in $y^{-1} \Phi(x)$ and $t y^{-1} \Phi(x)$ is the same. It follows that the contributions of $x y$ and $x s_{j} y$ to the series $F(\Phi ; \mathbf{q})$ cancel out, and thus the net contribution of the coset $W_{J} y$ is zero.

For perfect $y$, we still have (3), whence

$$
\sum_{x \in W_{J}} \operatorname{sgn}(x y) \prod_{\beta \in \Phi_{1}(x y)} q_{\beta}=\operatorname{sgn}(y) F\left(\Phi_{J} ; y^{-1} \mathbf{q}\right) \prod_{\beta \in \Phi_{1}(y)} q_{\beta}
$$

and the result follows.

Let $\rho^{\vee}$ be the unique co-weight such that $\left\langle\alpha_{i}, \rho^{\vee}\right\rangle=1$ for all $i \in I$. (It is wellknown that $2 \rho^{\vee}$ is also the sum of all positive co-roots.) We have found that the most convenient way to explicitly compute $G_{J}(\Phi ; \mathbf{q})$ under the coarse grading is as follows.

Proposition 5.4. Let $\Phi=\mathcal{O}_{1} \cup \dot{\cup} \mathcal{O}_{2} \cup$. . be the partition of $\Phi$ into $W$-orbits, and choose variables $q_{i}$ so that $q_{i}=q_{\beta}$ for all $\beta \in \mathcal{O}_{i} \cap \Phi_{1}^{+}$.

(a) The map $w \mapsto w \rho^{\vee}$ defines a bijection between the set ${ }^{J} W_{1}$ of perfect coset representatives and

$\left(W \rho^{\vee}\right)_{J}:=\left\{\theta \in W \rho^{\vee}:\left\langle\alpha_{j}, \theta\right\rangle\right.$ is odd and positive for all $\left.j \in J\right\}$.

(b) Under the coarse grading, we have

$$
\begin{gathered}
G_{J}(\Phi ; \mathbf{q})=\sum_{\theta \in\left(W \rho^{\vee}\right)_{J}}(-1)^{N(\theta)} q_{1}^{N_{1}(\theta)} q_{2}^{N_{2}(\theta)} \cdots, \\
\text { where } N(\theta):=\left|\left\{\beta \in \Phi^{+}:\langle\beta, \theta\rangle<0\right\}\right| \text { and } \\
N_{i}(\theta):=\mid\left\{\beta \in \mathcal{O}_{i} \cap \Phi^{+}:\langle\beta, \theta\rangle \text { is odd and negative }\right\} \mid .
\end{gathered}
$$

Note that the roots $\beta$ contributing to $N_{i}(\theta)$ may be odd or even.

Proof. (a) Recall that $w \in{ }^{J} W_{1}$ if and only if $w^{-1} \alpha_{j}$ is odd and positive for all $j \in J$. On the other hand, the height of a root $\beta$ is $\left\langle\beta, \rho^{\vee}\right\rangle$, so $\left\langle\alpha_{j}, w \rho^{\vee}\right\rangle=\left\langle w^{-1} \alpha_{j}, \rho^{\vee}\right\rangle$ is odd and positive if and only if $w^{-1} \alpha_{j}$ is odd and positive. Since $\rho^{\vee}$ is regular, the map $w \mapsto w \rho^{\vee}$ is injective, and the claim follows.

(b) Given $\theta=w \rho^{\vee}$ and a root $\beta$, we have $\langle\beta, \theta\rangle=\left\langle w^{-1} \beta, \rho^{\vee}\right\rangle$, so

$$
\Phi(w)=\left\{-w^{-1} \beta: \beta \in \Phi^{+},\langle\beta, \theta\rangle<0\right\}
$$

and $N(\theta)$ is the total number of inversions for $w$; i.e. $N(\theta)=\ell(w)$. Since $\beta$ and $-w^{-1} \beta$ clearly belong to the same $W$-orbit, and $-w^{-1} \beta$ is odd if and only if $\langle\beta, \theta\rangle$ is odd, we obtain that $N_{i}(\theta)$ is the number of odd inversions for $w$ in the $W$-orbit $\mathcal{O}_{i}$.

\section{REMARKS 5.5.}

(a) Determining the points in the $W$-orbit of $\rho^{\vee}$ that correspond to the perfect cosets for $W_{J} \backslash W$ does not require generating the entire orbit. Indeed, we claim that it is possible to efficiently generate the much smaller set ${ }^{J} W \rho^{\vee}$ consisting of members of the orbit that are $\Phi_{J}$-dominant, and select from these the ones that are perfect. Indeed, we claim that ${ }^{J} W \rho^{\vee}$ is the smallest set $X$ of co-weights that 
(i) contains $\rho^{\vee}$, and

(ii) contains $\theta-\beta^{\vee}$ if $\theta \in X, \beta \in \Phi^{+} \backslash \Phi_{J},\langle\beta, \theta\rangle=1$, and $\theta-\beta^{\vee}$ is $\Phi_{J}$-dominant.

Given that $\theta$ and $\beta$ satisfy the hypotheses for (ii), it follows that $\theta-\beta^{\vee}$ is the image of $\theta$ under the reflection corresponding to $\beta$. Thus the fact that ${ }^{J} W \rho^{\vee}$ contains $X$ follows by induction with respect to height. Conversely, given $w \in{ }^{J} W$, either $w=1$ and $w \rho^{\vee}=\rho^{\vee}$, or we have $\ell\left(w s_{i}\right)<\ell(w)$ and $\beta=-w \alpha_{i}>0$ for some $i$. We must also have $\beta \notin \Phi_{J}$, otherwise $w s_{i} w^{-1} \in W_{J}$ and $\ell\left(w s_{i}\right)=\ell\left(w s_{i} w^{-1} \cdot w\right)>\ell(w)$, a contradiction. Furthermore, it must be the case that $w s_{i} \in{ }^{J} W$. Otherwise, for some $j \in J$ we would have

$$
\ell\left(s_{j} w s_{i}\right)<\ell\left(w s_{i}\right)<\ell(w)<\ell\left(s_{j} w\right),
$$

contradicting the fact that $s_{j} w s_{i}$ and $s_{j} w$ differ in length by 1 . Thus by induction with respect to length, we may assume $w s_{i} \rho^{\vee} \in X$. However, $w s_{i} \rho^{\vee}=w\left(\rho^{\vee}-\alpha_{i}^{\vee}\right)=w \rho^{\vee}+\beta^{\vee}$ and $\left\langle\beta, w s_{i} \rho^{\vee}\right\rangle=\left\langle\alpha_{i}, \rho^{\vee}\right\rangle=1$, whence $w s_{i} \rho^{\vee}-\beta^{\vee}=w \rho^{\vee} \in X$ by (ii).

(b) For example, if $\left(\Phi, \Phi_{J}\right) \cong\left(E_{8}, E_{7}\right)$, then there are only 240 cosets for $W_{J} \backslash W$ (one for each root), and it is a fast calculation to generate the 240 vectors in ${ }^{J} W \rho^{\vee}$ by the above algorithm. It turns out that 32 of the 240 vectors correspond to cosets whose minimal representatives are perfect. In Table 2 , we list the co-weight coordinates of these 32 vectors; i.e. for each $w \in{ }^{J} W_{1}$, we list $\left\langle w \rho^{\vee}, \alpha_{i}\right\rangle$ for $1 \leqslant i \leqslant 8$, along with $\ell(w)=N\left(w \rho^{\vee}\right)$ and $\ell_{1}(w)=N_{1}\left(w \rho^{\vee}\right)$ (cf. the formulas in Proposition 5.4(b)).

Note that we have indexed the simple roots of $E_{8}$ as in this Dynkin diagram:

$$
\text { 1-3-4 } \stackrel{2}{\stackrel{2}{\mid}-5-6-7-8 .}
$$

The fact that the first 7 coordinates are odd and positive in each of the 32 cases confirms that these correspond to minimal coset representatives that are perfect.

\section{FACTORING THE FINE GRADED SERIES}

Following the road map laid out in the previous section, we are now in a position to explicitly evaluate the fine or coarse graded series for each irreducible root system. To set a convention for naming the variables for the coarse grading, let

- $q_{\beta}=q$ if $\Phi$ is of type $A, D$, or $E$,

- $q_{\beta}=p$ (if $\beta$ is short) or $q$ (if $\beta$ is long) for $B_{n}, F_{4}$, and $G_{2}$, and

- $q_{\beta}=q$ (if $\beta$ is short) or $r$ (if $\beta$ is long) for $C_{n}$

for all odd positive roots $\beta$. In this way, the variable attached to an odd root in $A_{n-1}$ is unchanged when $A_{n-1}$ is embedded as a parabolic subsystem of $B_{n}$ or $C_{n}$.

In types $A$ and $C$, where each $W$-orbit of roots contains two $W\left(\Phi_{0}\right)$-orbits of odd roots (Proposition 2.4), we will use the variables $q_{ \pm}$and $r_{ \pm}$for the fine grading. For $C_{n}$, the $W\left(\Phi_{0}\right)$-orbit containing the long simple root will be weighted $r_{+}$, and the short simple root that is adjacent to it in the Dynkin diagram will be weighted $q_{+}$. For $A_{2 n-1}$, the odd orbit containing the simple roots at both ends of the Dynkin diagram will be weighted $q_{+}$. For $A_{2 n}$, we have noted previously that the diagram automorphism interchanges the two $W\left(\Phi_{0}\right)$-orbits of odd roots, so the fine series is unaffected by the choice of which one is weighted $q_{+}$; either choice will suffice despite being non-canonical. 


\begin{tabular}{|cccccccr|c|c|}
\hline \multicolumn{6}{|c|}{$\left\langle w \rho^{\vee}, \alpha_{i}\right\rangle, i=1, \ldots, 8$} & $\ell(w)$ & $\ell_{1}(w)$ \\
\hline 1 & 1 & 1 & 1 & 1 & 1 & 1 & 1 & 0 & 0 \\
1 & 1 & 3 & 1 & 1 & 1 & 1 & -6 & 6 & 3 \\
1 & 1 & 1 & 3 & 1 & 1 & 1 & -10 & 10 & 5 \\
7 & 1 & 1 & 1 & 1 & 1 & 1 & -11 & 12 & 6 \\
1 & 1 & 3 & 1 & 1 & 3 & 1 & -14 & 14 & 7 \\
1 & 1 & 5 & 1 & 1 & 1 & 1 & -16 & 18 & 9 \\
3 & 1 & 1 & 1 & 1 & 5 & 1 & -19 & 20 & 10 \\
1 & 1 & 1 & 3 & 1 & 3 & 1 & -21 & 24 & 12 \\
1 & 1 & 1 & 3 & 1 & 1 & 5 & -25 & 28 & 14 \\
1 & 1 & 1 & 1 & 5 & 1 & 1 & -23 & 28 & 14 \\
1 & 5 & 1 & 1 & 1 & 3 & 1 & -23 & 28 & 14 \\
3 & 1 & 1 & 1 & 1 & 1 & 9 & -27 & 28 & 14 \\
1 & 1 & 3 & 1 & 1 & 1 & 7 & -26 & 28 & 15 \\
1 & 3 & 1 & 1 & 3 & 1 & 3 & -24 & 28 & 15 \\
1 & 1 & 1 & 1 & 1 & 1 & 11 & -28 & 28 & 15 \\
1 & 7 & 1 & 1 & 1 & 1 & 1 & -22 & 28 & 15 \\
\hline
\end{tabular}

\begin{tabular}{|cccccccc|c|c|}
\hline \multicolumn{7}{|c|}{$\left\langle w \rho^{\vee}, \alpha_{i}\right\rangle, i=1, \ldots, 8$} & $\ell(w)$ & $\ell_{1}(w)$ \\
\hline 1 & 1 & 3 & 1 & 1 & 1 & 7 & -27 & 29 & 14 \\
1 & 3 & 1 & 1 & 3 & 1 & 3 & -25 & 29 & 14 \\
1 & 1 & 1 & 1 & 1 & 1 & 11 & -29 & 29 & 14 \\
1 & 7 & 1 & 1 & 1 & 1 & 1 & -23 & 29 & 14 \\
1 & 1 & 1 & 3 & 1 & 1 & 5 & -26 & 29 & 15 \\
1 & 1 & 1 & 1 & 5 & 1 & 1 & -24 & 29 & 15 \\
1 & 5 & 1 & 1 & 1 & 3 & 1 & -24 & 29 & 15 \\
3 & 1 & 1 & 1 & 1 & 1 & 9 & -28 & 29 & 15 \\
1 & 1 & 1 & 3 & 1 & 3 & 1 & -26 & 33 & 17 \\
3 & 1 & 1 & 1 & 1 & 5 & 1 & -28 & 37 & 19 \\
1 & 1 & 5 & 1 & 1 & 1 & 1 & -27 & 39 & 20 \\
1 & 1 & 3 & 1 & 1 & 3 & 1 & -29 & 43 & 22 \\
7 & 1 & 1 & 1 & 1 & 1 & 1 & -28 & 45 & 23 \\
1 & 1 & 1 & 3 & 1 & 1 & 1 & -29 & 47 & 24 \\
1 & 1 & 3 & 1 & 1 & 1 & 1 & -29 & 51 & 26 \\
1 & 1 & 1 & 1 & 1 & 1 & 1 & -28 & 57 & 29 \\
\hline
\end{tabular}

TABle 2. Perfect coset representatives for $W\left(E_{7}\right) \backslash W\left(E_{8}\right)$.

For the fine series, it will be convenient to let $\mathbf{q} \rightarrow \overline{\mathbf{q}}$ denote the transformation of the variables in which the value of $q_{\beta}$ on a $W\left(\Phi_{0}\right)$-orbit $\mathcal{O}$ is replaced by its value on the opposite orbit $-\mathcal{O}$. For $A_{n}$ and $C_{n}$, this amounts to interchanging $q_{+}$with $q_{-}$ and $r_{+}$with $r_{-}$. In particular, as noted above,

$$
F\left(A_{2 n} ; \mathbf{q}\right)=F\left(A_{2 n} ; \overline{\mathbf{q}}\right) .
$$

Of course in the other irreducible cases, we know that every $W\left(\Phi_{0}\right)$-orbit is centrally symmetric $(\mathcal{O}=-\mathcal{O})$ and this transformation has no effect (Proposition 2.4).

In the following, we let $(a ; q)_{n}=(1-a)(1-a q) \cdots\left(1-a q^{n-1}\right)$.

TheOREm 6.1. For the finely graded series, we have

$$
\begin{aligned}
F\left(A_{2 n-1} ; \mathbf{q}\right) & =\left(1-q_{+}^{n}\right)\left(q_{+} q_{-} ; q_{+} q_{-}\right)_{n-1}, \\
F\left(A_{2 n} ; \mathbf{q}\right) & =\left(q_{+} q_{-} ; q_{+} q_{-}\right)_{n}, \\
F\left(B_{2 n} ; \mathbf{q}\right) & =\left(q^{2} ; q^{2}\right)_{n}\left(p ; q^{2}\right)_{n} \\
F\left(B_{2 n+1} ; \mathbf{q}\right) & =\left(q^{2} ; q^{2}\right)_{n}\left(p ; q^{2}\right)_{n+1}, \\
F\left(C_{2 n} ; \mathbf{q}\right) & =\left(1-q_{+}^{n}\right)\left(q_{+} q_{-} ; q_{+} q_{-}\right)_{n-1}\left(r_{+} r_{-} ; q_{+} q_{-}\right)_{n}, \\
F\left(C_{2 n+1} ; \mathbf{q}\right) & =\left(1-r_{+} q_{-}^{n}\right)\left(q_{+} q_{-} ; q_{+} q_{-}\right)_{n}\left(r_{+} r_{-} ; q_{+} q_{-}\right)_{n}, \\
F\left(D_{2 n} ; \mathbf{q}\right) & =\left(1-q^{n}\right)^{2}\left(q^{2} ; q^{2}\right)_{n-1}^{2}, \\
F\left(D_{2 n+1} ; \mathbf{q}\right) & =\left(q^{2} ; q^{2}\right)_{n}^{2} \\
F\left(E_{6} ; \mathbf{q}\right) & =\left(q^{2} ; q^{2}\right)_{4}, \\
F\left(E_{7} ; \mathbf{q}\right) & =\left(q^{2} ; q\right)_{7}, \\
F\left(E_{8} ; \mathbf{q}\right) & =\left(1-q^{8}\right)\left(q^{2} ; q^{2}\right)_{7}, \\
F\left(F_{4} ; \mathbf{q}\right) & =\left(1-p^{2}\right)\left(1-q^{2}\right)\left(1-p^{2} q^{2}\right)\left(1-p^{2} q^{4}\right), \\
F\left(G_{2} ; \mathbf{q}\right) & =\left(1-p^{2}\right)\left(1-q^{2}\right) .
\end{aligned}
$$


Proof. Proceed by induction with respect to rank. In most cases, the claimed identity may be proved by using Proposition 5.4 to compute the series $G_{J}(\Phi ; \mathbf{q})$ for a suitable parabolic subsystem $\Phi_{J}$ of co-rank 1 , and then appealing to Corollary 5.2 and the induction hypothesis. However, for $A_{n}$ and $C_{n}$, our induction will directly rely on Theorem 5.1.

CASE 1: $\Phi=A_{n}$. With coordinates chosen so that $\alpha_{i}=\varepsilon_{i+1}-\varepsilon_{i}(1 \leqslant i \leqslant n)$, the co-weight $\rho^{\vee}$ is the orthogonal projection of $(0,1, \ldots, n)$ into the hyperplane normal to $(1, \ldots, 1)$. The $W$-orbit of $\rho^{\vee}$ may thus be identified with the permutations of $\{0, \ldots, n\}$. If $\Phi_{J} \cong A_{n-1}$ omits the first simple root, then the members of this orbit that are $\Phi_{J}$-dominant are of the form $\left(a_{0}, \ldots, a_{n}\right)$ with $a_{1}<\cdots<a_{n}$, and the ones that correspond to perfect cosets (see Proposition 5.4(a)) must also have $a_{i+1}-$ $a_{i}$ odd for all $i \geqslant 1$. Only two permutations satisfy this property: $(0,1, \ldots, n)$ and $(n, 0, \ldots, n-1)$. Thus there are only two perfect coset representatives: the identity element and $y=s_{1} s_{2} \ldots s_{n}$. Of course $y$ has $n$ inversions; the odd ones are the roots $\varepsilon_{n+1}-\varepsilon_{i}=\alpha_{n}+\cdots+\alpha_{i}$ for $i=n \bmod 2$. These all belong to the $W\left(\Phi_{0}\right)$-orbit of $\alpha_{n}$. If $n$ is odd, then these inversions are all weighted $q_{+}$, and Corollary 5.3 yields

$$
F\left(A_{2 n-1} ; \mathbf{q}\right)=\left(1-q_{+}^{n}\right) F\left(A_{2 n-2} ; \mathbf{q}\right) .
$$

For even $n$, there is no harm in declaring the odd root $\alpha_{n}$ to have weight $q_{+}$(recall (4)). This agrees with the weight it is assigned as a member of $\Phi_{J}$. However, it is the opposite of the weight assigned to $y^{-1} \alpha_{n}=\alpha_{n-1}$, so the effect of the substitution $\mathbf{q} \rightarrow y^{-1} \mathbf{q}$ on $F\left(\Phi_{J} ; \mathbf{q}\right)$ is the change of variables $\mathbf{q} \rightarrow \overline{\mathbf{q}}$. Thus Theorem 5.1 in this case implies

$$
\begin{aligned}
F\left(A_{2 n} ; \mathbf{q}\right) & =F\left(A_{2 n-1} ; \mathbf{q}\right)+q_{+}^{n} F\left(A_{2 n-1} ; \overline{\mathbf{q}}\right) \\
& =\left(1-q_{+}^{n}\right) F\left(A_{2 n-2} ; \mathbf{q}\right)+q_{+}^{n}\left(1-q_{-}^{n}\right) F\left(A_{2 n-2} ; \mathbf{q}\right) \\
& =\left(1-q_{+}^{n} q_{-}^{n}\right) F\left(A_{2 n-2} ; \mathbf{q}\right),
\end{aligned}
$$

the second equality being a consequence of (4) and (5).

CASE 2: $\Phi=B_{n}$. By choosing simple roots $\alpha_{1}=\varepsilon_{1}$ and $\alpha_{i}=\varepsilon_{i}-\varepsilon_{i-1}(1<i \leqslant n)$, we have $\rho^{\vee}=(1,2, \ldots, n)$. The $W$-orbit of $\rho^{\vee}$ consists of all signed permutations of $\{1, \ldots, n\}$, and with $\Phi_{J} \cong B_{n-1}$ omitting the simple root $\alpha_{n}$, one sees that a member $\left(a_{1}, \ldots, a_{n}\right)$ of this orbit is $\Phi_{J}$-dominant when $0<a_{1}<\cdots<a_{n-1}$. Imposing the additional constraints in Proposition 5.4(a) (i.e. $a_{i}=i \bmod 2$ ), we see that there is only one nontrivial member of this orbit that corresponds to a perfect coset; namely, $\theta=(1, \ldots, n-1,-n)$. Since

$$
\left\{\beta \in \Phi^{+}:\langle\beta, \theta\rangle<0\right\}=\left\{\varepsilon_{n}\right\} \cup\left\{\varepsilon_{n} \pm \varepsilon_{i}: i<n\right\},
$$

one infers that this coset representative has $2 n-1$ inversions. Among these roots, we have $\langle\beta, \theta\rangle$ odd if and only if $\beta=\varepsilon_{n}$ and $n$ is odd, or $\beta=\varepsilon_{n} \pm \varepsilon_{i}$ and $i \neq n \bmod 2$. Thus Proposition 5.4 and Corollary 5.2 imply

$$
F\left(B_{n} ; \mathbf{q}\right) / F\left(B_{n-1} ; \mathbf{q}\right)= \begin{cases}1-p q^{n-1} & \text { if } n \text { is odd } \\ 1-q^{n} & \text { if } n \text { is even }\end{cases}
$$

For the base of this induction, one may specialize the series for $C_{2}$.

CASE 3: $\Phi=C_{n}$. With simple roots $\alpha_{1}=2 \varepsilon_{1}$ and $\alpha_{i}=\varepsilon_{i}-\varepsilon_{i-1}(1<i \leqslant n)$, one has $2 \rho^{\vee}=(1,3, \ldots, 2 n-1)$. The $W$-orbit of $2 \rho^{\vee}$ consists of all signed permutations of these coordinates, and with $J$ chosen so that $\Phi_{J} \cong C_{n-1}$, the condition for a member $\left(a_{1}, \ldots, a_{n}\right)$ of this orbit to be $\Phi_{J}$-dominant is $0<a_{1}<\cdots<a_{n-1}$. After accounting 
for the extra factor of 2, the perfection constraints of Proposition 5.4(a) amount to the added condition that $a_{i}-a_{i-1}=2 \bmod 4$ for $1<i<n$, leaving four possibilities:

$$
(1,3, \ldots, 2 n-3, \pm(2 n-1)), \quad(3,5, \ldots, 2 n-1, \pm 1) .
$$

The corresponding perfect coset representatives are

$$
{ }^{J} W_{1}=\left\{1, y, y s_{1}, y s_{1} y^{-1}\right\},
$$

where $y=s_{n} \cdots s_{3} s_{2}$. Thus there are four terms in the recurrence for $F\left(C_{n} ; \mathbf{q}\right)$ provided by Theorem 5.1. Of course the term contributed by the identity element is $F\left(C_{n-1} ; \mathbf{q}\right)$. For the remaining three, the inversion sets are

$$
\begin{gathered}
\Phi(y)=\left\{\varepsilon_{i}-\varepsilon_{1}: 1<i \leqslant n\right\}, \quad \Phi\left(y s_{1}\right)=\left\{\varepsilon_{i}+\varepsilon_{1}: 1 \leqslant i \leqslant n\right\}, \\
\Phi\left(y s_{1} y^{-1}\right)=\Phi^{+} \backslash \Phi_{J}=\left\{2 \varepsilon_{n}\right\} \cup\left\{\varepsilon_{n} \pm \varepsilon_{i}: 1 \leqslant i<n\right\} .
\end{gathered}
$$

Now recall from the proof of Proposition 2.4 that $\beta \in C_{n}$ is odd if and only if $\langle\beta, \omega\rangle= \pm 2$, where $\omega=(1,-1,1,-1, \ldots)$. Moreover, our conventions for the variables $q_{\beta}$ are such that if $\langle\beta, \omega\rangle=2$, then $q_{\beta}=r_{+}$(if $\beta$ is long) or $q_{-}$(if $\beta$ is short).

Hence the odd inversions for $y s_{1} y^{-1}$ are $\varepsilon_{n}+\varepsilon_{i}($ for $i=n \bmod 2), \varepsilon_{n}-\varepsilon_{i}$ (for $i \neq n \bmod 2$ ), and $2 \varepsilon_{n}$. These have weights $q_{+}$and $r_{-}$for $n$ even, or $q_{-}$and $r_{+}$ for $n$ odd. Since $y s_{1} y^{-1}$ acts trivially on $\Phi_{J}$, we conclude that this term contributes $-r_{-} q_{+}^{n-1} F\left(C_{n-1} ; \mathbf{q}\right)$ for $n$ even or $-r_{+} q_{-}^{n-1} F\left(C_{n-1} ; \mathbf{q}\right)$ for $n$ odd.

Similarly, the odd inversions for $y$ are $\varepsilon_{2 i}-\varepsilon_{1}$ (all of weight $q_{+}$), and for $y s_{1}$ they are $2 \varepsilon_{1}$ (of weight $r_{+}$) and $\varepsilon_{2 i+1}+\varepsilon_{1}$ (of weight $q_{-}$). Furthermore, $y^{-1} \alpha_{1}=\left(y s_{1}\right)^{-1} \alpha_{1}=$ $2 \varepsilon_{2}$ and $y^{-1} \alpha_{2}=\left(y s_{1}\right)^{-1} \alpha_{2}=\alpha_{3}$, so the substitutions $\mathbf{q} \rightarrow y^{-1} \mathbf{q}$ and $\mathbf{q} \rightarrow\left(y s_{1}\right)^{-1} \mathbf{q}$ are equivalent to the change of variable $\mathbf{q} \rightarrow \overline{\mathbf{q}}$. Thus Theorem 5.1 implies

$$
\begin{aligned}
F\left(C_{2 n} ; \mathbf{q}\right) & =\left(1-r_{-} q_{+}^{2 n-1}\right) F\left(C_{2 n-1} ; \mathbf{q}\right)+\left(r_{+} q_{-}^{n-1}-q_{+}^{n}\right) F\left(C_{2 n-1} ; \overline{\mathbf{q}}\right), \\
F\left(C_{2 n+1} ; \mathbf{q}\right) & =\left(1-r_{+} q_{-}^{2 n}\right) F\left(C_{2 n} ; \mathbf{q}\right)-\left(r_{+} q_{-}^{n}-q_{+}^{n}\right) F\left(C_{2 n} ; \overline{\mathbf{q}}\right) .
\end{aligned}
$$

After isolating common factors, one sees that having the claimed product formula for $F\left(C_{n} ; \mathbf{q}\right)$ satisfy the above recurrence reduces to confirming the pair of identities

$$
\begin{aligned}
&\left(1-q_{+}^{n}\right)\left(1-r_{+} r_{-} q_{+}^{n-1} q_{-}^{n-1}\right)=\left(1-r_{-} q_{+}^{2 n-1}\right)\left(1-r_{+} q_{-}^{n-1}\right) \\
&+\left(r_{+} q_{-}^{n-1}-q_{+}^{n}\right)\left(1-r_{-} q_{+}^{n-1}\right), \\
&\left(1-r_{+} q_{-}^{n}\right)\left(1-q_{+}^{n} q_{-}^{n}\right)=\left(1-r_{+} q_{-}^{2 n}\right)\left(1-q_{+}^{n}\right)-\left(r_{+} q_{-}^{n}-q_{+}^{n}\right)\left(1-q_{-}^{n}\right) .
\end{aligned}
$$

After checking directly that $F\left(C_{2} ; \mathbf{q}\right)=\left(1-q_{+}\right)\left(1-r_{+} r_{-}\right)$, this induction is complete.

CASE 4: $\Phi=D_{n}$. With simple roots $\alpha_{1}=\varepsilon_{1}+\varepsilon_{2}$ and $\alpha_{i}=\varepsilon_{i}-\varepsilon_{i-1}(1<i \leqslant n)$, one has $\rho^{\vee}=(0,1, \ldots, n-1)$. The $W$-orbit of $\rho^{\vee}$ consists of all signed permutations of these coordinates, and with $\Phi_{J} \cong D_{n-1}$ omitting the simple root $\alpha_{n}$, one sees that a member $\left(a_{1}, \ldots, a_{n}\right)$ of this orbit is $\Phi_{J}$-dominant when $\left|a_{1}\right|<a_{2}<\cdots<a_{n-1}$. The perfection constraints in Proposition 5.4(a) require $a_{i}-a_{i-1}$ to be odd for $1<i<n$, yielding four points in $\left(W \rho^{\vee}\right)_{J}$; namely,

$$
\theta_{ \pm 1}=( \pm 1,2, \ldots, n-1,0), \quad \theta_{ \pm n}=(0,1, \ldots, n-2, \pm(n-1)) .
$$

Of course $\theta_{n}=\rho^{\vee}$ contributes 1 to $G_{J}(\Phi ; \mathbf{q})$. In the remaining cases, the roots in $\Phi^{+} \backslash \Phi_{J}$ are of the form $\varepsilon_{n} \pm \varepsilon_{i}$ for $i<n$, and we have

$$
\left\langle\varepsilon_{n} \pm \varepsilon_{i}, \theta_{1}\right\rangle= \pm i, \quad\left\langle\varepsilon_{n} \pm \varepsilon_{i}, \theta_{-n}\right\rangle=-(n-1) \pm(i-1) .
$$

The results for $\theta_{-1}$ are similar to $\theta_{1}$ except that the values for $\varepsilon_{n} \pm \varepsilon_{1}$ are swapped. Thus the coset representatives corresponding to $\theta_{ \pm 1}$ and $\theta_{-n}$ have $n-1$ and $2(n-1)$ inversions, respectively. The negative odd evaluations for $\theta_{ \pm 1}$ occur once for each 
odd $i$, and for $\theta_{-n}$ they occur twice for each $i \neq n \bmod 2$. Thus Proposition 5.4 and Corollary 5.2 imply

$$
F\left(D_{2 n} ; \mathbf{q}\right) / F\left(D_{2 n-1} ; \mathbf{q}\right)=\left(1-q^{n}\right)^{2}, \quad F\left(D_{2 n+1} ; \mathbf{q}\right) / F\left(D_{2 n} ; \mathbf{q}\right)=\left(1+q^{n}\right)^{2} .
$$

For the base of this induction, one may use the univariate series for $A_{3} \cong D_{3}$.

CASE 5. The remaining possibilities are the five exceptional root systems. For $G_{2}$, it is probably easiest to evaluate the series directly either via Proposition 4.1 or the definition. We leave this to the reader. For $\Phi=F_{4}, E_{6}, E_{7}$, and $E_{8}$, we will give a high-level description of the computation.

First, we choose parabolic subsystems $\Phi_{J}$ isomorphic to $B_{3}, D_{5}, E_{6}$, and $E_{7}$ (respectively), and generate the $\Phi_{J}$-dominant portion of the $W$-orbit of $\rho^{\vee}$ via the algorithm in Remark 5.5. The respective sizes of these sets are 24, 27, 56, and 240.

It is then an easy computation to select from these sets the vectors $\theta=w \rho^{\vee}$ that satisfy the perfection constraints of Proposition 5.4(a) (i.e. $\theta$ such that $\left\langle\theta, \alpha_{j}\right\rangle$ is odd and positive for all $j \in J)$. One obtains $6,6,8$, and 32 such vectors, respectively.

Coordinates for the 32 vectors arising in the case of $E_{8}$ were provided in Table 2.

Once the vectors have been collected, it is a routine application of Proposition 5.4 and Corollary 5.2 to compute $F(\Phi ; \mathbf{q}) / F\left(\Phi_{J} ; \mathbf{q}\right)$. One obtains

$$
\begin{aligned}
& F\left(F_{4} ; \mathbf{q}\right) / F\left(B_{3} ; \mathbf{q}\right)=(1+p)\left(1+p q^{2}\right)\left(1-p^{2} q^{2}\right), \\
& F\left(E_{6} ; \mathbf{q}\right) / F\left(D_{5} ; \mathbf{q}\right)=\left(1+q^{4}\right)\left(1+q^{2}+q^{4}\right), \\
& F\left(E_{7} ; \mathbf{q}\right) / F\left(E_{6} ; \mathbf{q}\right)=\left(1-q^{3}\right)\left(1-q^{5}\right)\left(1-q^{7}\right), \\
& F\left(E_{8} ; \mathbf{q}\right) / F\left(E_{7} ; \mathbf{q}\right)=\left(1-q^{8}\right)\left(1+q^{3}\right)\left(1+q^{5}\right)\left(1+q^{6}\right)\left(1+q^{7}\right) .
\end{aligned}
$$

REMARK 6.2. As mentioned in the introduction, the univariate series $F(\Phi ; q)$ except for the case $\Phi=E_{8}$ were determined previously by Brenti and Carnevale in [3] (see also the references cited there for earlier work in special cases). It is interesting to note that they give multivariate refinements of the coarse graded series for $B_{n}$ and $C_{n}$ that use 3 and 4 variables, respectively. (See Theorem 5.5 and the first formula in Theorem 5.7 in [3].) Curiously, their 4 variable series for $C_{n}$ and our fine graded series do not record the same information about the distribution of odd inversions.

\section{The PARABOliC SERIES}

We now turn our attention to the parabolic series $F_{J}(\Phi ; \mathbf{q})$ introduced in Section 4. We will say that a subsystem $\Phi_{J} \subset \Phi$ is coherent whenever

$$
F_{J}(\Phi ; \mathbf{q})=F(\Phi ; \mathbf{q}) / F\left(\Phi_{J} ; \mathbf{q}\right)
$$

under the fine grading. Since we have explicit formulas for the full series, we therefore have explicit formulas for $F_{J}(\Phi ; \mathbf{q})$ whenever $\Phi_{J}$ is coherent.

Of course it cannot generally be the case that all subsystems are coherent. For example, we have previously noted that the quotient $F(\Phi ; \mathbf{q}) / F\left(\Phi_{J} ; \mathbf{q}\right)$ need not even be a polynomial in types $A$ and $C$. If we restrict to the coarse or univariate gradings, this obstruction is removed (Corollary 5.2); however, we have also seen that the two sides of (6) need not even have the same degree (Remark 4.3).

On the other hand, we claim that there is a large class of coherent parabolic subsystems. To describe this class, consider that the height functions in $\Phi$ and $\Phi_{J}$ are compatible, so $\Phi_{J}$ has an even-height root subsystem $\Phi_{J, 0} \subseteq \Phi_{0}$, an even Weyl group $W\left(\Phi_{J, 0}\right)=W_{J} \cap W\left(\Phi_{0}\right)$, and a $W_{J}$-normalizer $N\left(\Phi_{J, 0}\right)=W\left(\Phi_{J, 0}\right) \rtimes \Gamma\left(\Phi_{J, 0}\right)$. As noted previously, $\Gamma\left(\Phi_{0}\right)$ may be identified as the subgroup of $N\left(\Phi_{0}\right)$ consisting of elements with no even inversions, so there is an inclusion $N\left(\Phi_{J, 0}\right) \subseteq N\left(\Phi_{0}\right)$ if and 
only if $\Gamma\left(\Phi_{J, 0}\right) \subseteq \Gamma\left(\Phi_{0}\right)$. Moreover, one may test for this inclusion merely by checking that $y \alpha_{j}$ is odd for all $y \in \Gamma\left(\Phi_{J, 0}\right)$ and all $j \notin J$.

THEOREM 7.1. If $\Gamma\left(\Phi_{J, 0}\right) \subseteq \Gamma\left(\Phi_{0}\right)$ (or equivalently, $N\left(\Phi_{J, 0}\right) \subseteq N\left(\Phi_{0}\right)$ ), then $\Phi_{J}$ is coherent. That is, we have $F_{J}(\Phi ; \mathbf{q})=F(\Phi ; \mathbf{q}) / F\left(\Phi_{J} ; \mathbf{q}\right)$ under the fine grading.

We know that in many cases, $F(\Phi ; \mathbf{q}) / F\left(\Phi_{J} ; \mathbf{q}\right)$ is $G_{J}(\Phi ; \mathbf{q})$, the sign-twisted series for the set ${ }^{J} W_{1}$ of perfect coset representatives (Theorem 5.1). Thus one available strategy for proving coherence in some cases would be to show $F_{J}(\Phi ; \mathbf{q})=G_{J}(\Phi ; \mathbf{q})$ directly.

LEMMA 7.2. If ${ }^{J} W_{1} \subset W\left(\Phi_{0}\right)$, then $F(\Phi ; \mathbf{q}) / F\left(\Phi_{J} ; \mathbf{q}\right)=G_{J}(\Phi ; \mathbf{q})=F_{J}(\Phi ; \overline{\mathbf{q}})$ under the fine grading.

Proof. It is clear that $F\left(\Phi_{J} ; \mathbf{q}\right)=F\left(\Phi_{J} ; y^{-1} \mathbf{q}\right)$ for any $y \in{ }^{J} W \cap W\left(\Phi_{0}\right)$, so the first equality is an immediate consequence of Theorem 5.1 . For the second, note that

$$
{ }^{J} W_{1} \subseteq W\left(\Phi_{0}\right) \cap{ }^{J} W \subseteq N\left(\Phi_{0}\right) \cap{ }^{J} W .
$$

On the other hand, any $y \in N\left(\Phi_{0}\right)$ has the property that $y^{-1} \alpha_{i}$ is odd for all $i$, and thus equality occurs throughout. We therefore have

$$
G_{J}(\Phi ; \mathbf{q})=\sum_{y \in N\left(\Phi_{0}\right) \cap^{J} W} \operatorname{sgn}(y) \prod_{\beta \in \Phi_{1}(y)} q_{\beta}=\sum_{y \in N\left(\Phi_{0}\right) \cap W^{J}} \operatorname{sgn}(y) \prod_{\beta \in \Phi_{1}\left(y^{-1}\right)} q_{\beta} .
$$

The only difference between the second sum and the expression for $F_{J}(\Phi ; \mathbf{q})$ in Proposition 4.1 is the dependence on the inversion set for $y^{-1}$ rather than $y$. However, the conditions for $\beta$ to be an inversion for $y$ (i.e. $\beta>0$ and $y \beta<0$ ) are also equivalent to having $-y \beta$ be an inversion for $y^{-1}$. Moreover, $\beta$ is odd if and only if $-y \beta$ is odd, and the two roots necessarily belong to opposite $W\left(\Phi_{0}\right)$-orbits (since $y \in W\left(\Phi_{0}\right)$ ). Thus the above sum is identical to the result of substituting $\mathbf{q} \rightarrow \overline{\mathbf{q}}$ in Proposition 4.1.

Proof of Theorem 7.1. By definition, we have

$$
F(\Phi ; \mathbf{q})=\sum_{x \in W^{J}} \sum_{y \in W_{J}} \operatorname{sgn}(x y) \prod_{\beta \in \Phi_{1}(x y)} q_{\beta} .
$$

By Proposition 4.1, this sum may be restricted to those pairs $(x, y)$ with $x y \in N\left(\Phi_{0}\right)$.

Applying Lemma 4.2 to the pair $\left(\Phi_{J}, \Phi_{\varnothing}\right)$, we know that the map $y \mapsto s_{j} y$ (with $j$ being the first fault of $y$ relative to $\Phi_{J}$ ) is a sign-reversing involution on the members of $W_{J}$ not in $N\left(\Phi_{J, 0}\right)$. Replacing $y$ with $s_{j} y$ if necessary, we may assume $\ell\left(s_{j} y\right)>\ell(y)$. Since $\ell(x y)=\ell(x)+\ell(y)$ for all $x \in W^{J}$ and $y \in W_{J}$, Lemma 1.1 implies

$$
\begin{aligned}
\Phi(x y) & =\Phi(y) \dot{\cup} y^{-1} \Phi(x), \\
\Phi\left(x s_{j} y\right) & =\Phi(y) \dot{\cup}\left\{y^{-1} \alpha_{j}\right\} \dot{\cup} y^{-1} s_{j} \Phi(x) \\
& =\Phi(y) \dot{\cup}\left\{y^{-1} \alpha_{j}\right\} \dot{\cup} t y^{-1} \Phi(x),
\end{aligned}
$$

where $t=y^{-1} s_{j} y$ is the reflection corresponding to the root $y^{-1} \alpha_{j} \in \Phi_{J}$. However, $j$ is the first fault of $y$ relative to $\Phi_{J}$, so this is an even root and $t$ belongs to $W\left(\Phi_{J, 0}\right) \subset$ $W\left(\Phi_{0}\right)$. It follows that for each $W\left(\Phi_{0}\right)$-orbit of odd roots, the number of such roots is the same in $y^{-1} \Phi(x)$ and $t y^{-1} \Phi(x)$. Furthermore, $N\left(\Phi_{0}\right)$ includes $x s_{j} y=x y t$ if and only if it also includes $x y$. Thus the contributions of $x y$ and $x s_{j} y$ to (7) cancel out under the fine grading, and we can require $y \in N\left(\Phi_{J, 0}\right)$ in addition to the restriction $x y \in N\left(\Phi_{0}\right)$. 
Given the hypothesis $N\left(\Phi_{J, 0}\right) \subset N\left(\Phi_{0}\right)$, our restrictions on $x$ and $y$ can be separated into $x \in N\left(\Phi_{0}\right), y \in N\left(\Phi_{J, 0}\right)$. Since $\Phi_{1}(x y)=\Phi_{1}(y) \dot{\cup} y^{-1} \Phi_{1}(x)$, we obtain

$$
\begin{aligned}
F(\Phi ; \mathbf{q}) & =\sum_{x \in N\left(\Phi_{0}\right) \cap W^{J}} \sum_{y \in N\left(\Phi_{J, 0}\right)} \operatorname{sgn}(x y) \prod_{\beta \in \Phi_{1}(x y)} q_{\beta} \\
& =\sum_{y \in N\left(\Phi_{J, 0}\right)}\left(\operatorname{sgn}(y) \prod_{\beta \in \Phi_{1}(y)} q_{\beta}\right) F_{J}\left(\Phi ; y^{-1} \mathbf{q}\right)
\end{aligned}
$$

under the fine grading. It therefore suffices to show that

$$
F_{J}(\Phi ; \mathbf{q})=F_{J}\left(\Phi ; y^{-1} \mathbf{q}\right) \text { for all } y \in N\left(\Phi_{J, 0}\right)
$$

Indeed, in that case we could factor $F_{J}(\Phi ; \mathbf{q})$ out of the above sum, and Proposition 4.1 would clearly identify the remaining sum as $F\left(\Phi_{J} ; \mathbf{q}\right)$.

This invariance is immediate whenever $y$ belongs to $W\left(\Phi_{J, 0}\right.$ ) (a subgroup of $\left.W\left(\Phi_{0}\right)\right)$, so we may assume $y \in \Gamma\left(\Phi_{J, 0}\right) \subseteq \Gamma\left(\Phi_{0}\right)$ and that $y$ acts nontrivially on the $W\left(\Phi_{0}\right)$-orbits of odd roots. We may also assume $\Phi_{J}$ is proper (otherwise $W^{J}$ is trivial) and that $y \in \Gamma\left(\Phi_{K, 0}\right)$ for some irreducible component $\Phi_{K}$ of $\Phi_{J}$, since $\Gamma\left(\Phi_{J, 0}\right)$ is generated by such groups. In that case, $y$ may act non-trivially on the orbits of odd roots in at most one irreducible component of $\Phi$ (the one containing $\Phi_{K}$ ), so we may further assume that $\Phi$ is irreducible. By Proposition 3.2, this is possible only if $\Phi \cong A_{2 n-1}$ or $C_{n}$.

In these cases, there is only one nontrivial element $y \in \Gamma\left(\Phi_{0}\right)$, and Proposition 3.2 provides an explicit description of it. For $A_{2 n-1}$ and $C_{2 n-1}$, it involves the simple reflections at both ends of the Dynkin diagram, and thus cannot belong to any proper parabolic subgroup of $W$ that is irreducible. For $C_{2 n}$, it is also the nontrivial element of $\Gamma\left(\Phi_{J, 0}\right)$, where $\Phi_{J}$ is the parabolic subsystem spanned by the short simple roots. Thus the conditions of the previous paragraph may be met only if $\left(\Phi, \Phi_{J}\right) \cong\left(C_{2 n}, A_{2 n-1}\right)$. Since $\Gamma\left(\Phi_{0}\right)$ acts on the $W\left(\Phi_{0}\right)$-orbits by interchanging opposite pairs (Proposition 3.2 again), proving

$$
F_{J}(\Phi ; \mathbf{q})=F_{J}(\Phi ; \overline{\mathbf{q}})
$$

in this one case completes the proof of (8).

Our strategy for this case is to show that the conditions of Lemma 7.2 are met; i.e. that $W\left(\Phi_{0}\right)$ contains all of the perfect coset representatives. The key point is that the long roots of $C_{2 n}$ are all odd. Thus every $y \in{ }^{J} W_{1}$ has the property that $y^{-1} \alpha_{i}$ is odd, whether $\alpha_{i}$ is short (i.e. $i \in J$ ) or $\alpha_{i}$ is long (i.e. $i \notin J$ ). In other words, ${ }^{J} W_{1} \subset N\left(\Phi_{0}\right)$, and the only question is whether ${ }^{J} W_{1}$ (or the entirety of ${ }^{J} W$ ) contains any elements in the nontrivial coset of $N\left(\Phi_{0}\right) / W\left(\Phi_{0}\right)$. For this, consider any $y \in{ }^{J} W$. Using the coordinates for $C_{2 n}$ we introduced in Section 2, we claim that either $y \varepsilon_{2 n}=\varepsilon_{2 n}$ or $y \varepsilon_{2 n}=-\varepsilon_{1}$. Otherwise, either $y \varepsilon_{2 n}=\varepsilon_{i}$ for some $i<2 n$ or $y \varepsilon_{2 n}=-\varepsilon_{i}$ for some $i>1$. In the former case, we have $y^{-1}\left(\varepsilon_{i+1}-\varepsilon_{i}\right)= \pm \varepsilon_{j}-\varepsilon_{2 n}<0$ for some $j$, and in the latter case, we have $y^{-1}\left(\varepsilon_{i}-\varepsilon_{i-1}\right)=-\varepsilon_{2 n} \pm \varepsilon_{j}<0$ for some $j$. Either way, we contradict the fact that $y \in{ }^{J} W$ and the claim follows. Thus $y$ maps the (odd) root $2 \varepsilon_{2 n}$ to either $2 \varepsilon_{2 n}$ or $-2 \varepsilon_{1}$. Both roots belong to the same $W\left(\Phi_{0}\right)$-orbit, so this confirms that every $y \in{ }^{J} W_{1}$ belongs to $W\left(\Phi_{0}\right)$. Thus Lemma 7.2 implies

$$
F_{J}(\Phi ; \overline{\mathbf{q}})=F(\Phi ; \mathbf{q}) / F\left(\Phi_{J} ; \mathbf{q}\right)=F\left(C_{2 n} ; \mathbf{q}\right) / F\left(A_{2 n-1} ; \mathbf{q}\right)
$$

under the fine grading. However, one can see from Theorem 6.1 that the right hand side is invariant under $\mathbf{q} \rightarrow \overline{\mathbf{q}}$, so the same is true for $F_{J}(\Phi ; \mathbf{q})$ and (9) follows. 


\section{REMARKS 7.3.}

(a) The irreducible root systems for which $\Gamma\left(\Phi_{0}\right)$ is trivial are $A_{2 n}, E_{6}, E_{8}, F_{4}$, and $G_{2}$ (see Table 1 ). Thus the conditions of Theorem 7.1 are easily met whenever the irreducible components of $\Phi_{J}$ are on this list. Conversely, if $\Phi$ is on this list, then this is the only way to meet the conditions.

(b) It follows in particular that the parabolic subsystems of $A_{2 n}$ whose irreducible components all have even rank are coherent. Conversely, it is easy to see from Theorem 6.1 that there are no other parabolic subsystems of $A_{2 n}$ with this property. Indeed, these are the only cases for which $F\left(\Phi_{J} ; \mathbf{q}\right)$ is a divisor of $F(\Phi ; \mathbf{q})$.

(c) In the case $\Phi=B_{n}$, the nontrivial element in $\Gamma\left(\Phi_{0}\right)$ is the short simple reflection. Thus Theorem 7.1 in this case implies that a parabolic subsystem of $B_{n}$ is coherent if each of its irreducible components contain either the short simple root or an even number of long simple roots.

(d) It seems likely that the converse of Theorem 7.1 is true; i.e. $\Phi_{J}$ is coherent if and only if $\Gamma\left(\Phi_{J, 0}\right) \subseteq \Gamma\left(\Phi_{0}\right)$. We have confirmed this for all $\Phi$ of rank at most 8, and the argument in (b) confirms it for $A_{2 n}$.

It appears that there is potential for a very general factorization theorem involving nearly all of the parabolic series $F_{J}(\Phi ; \mathbf{q})$. For example, Brenti and Carnevale have proved explicit product formulas for all of the univariate parabolic series $F_{J}\left(A_{n} ; q\right)$ and $F_{J}\left(B_{n} ; q\right)$ (see Corollary 4.3 and Theorem 5.4 in [4]); the latter was also obtained independently by Landesman [8]. These formulas are clearly expressible as products of cyclotomic polynomials, so they support the following:

CONJECTURE 7.4. If $\Phi$ is irreducible, then the univariate series $F_{J}(\Phi ; q)$ is a product of cyclotomic polynomials unless

(a) $\Phi \cong D_{2 n}$ and $I \supsetneq J \supseteq K$, where $\Phi_{K} \cong A_{1}^{\oplus n+1}$, or

(b) $\Phi \cong E_{7}$ and $I \supsetneq J \supseteq K$, where $\Phi_{K} \cong A_{1} \oplus D_{4}$, or

(c) $\Phi \cong E_{8}$ and $I \supsetneq J \supseteq K$, where $\Phi_{K} \cong D_{6}$.

Note that in each of (a)-(c), the subset $K \subset I$ is uniquely determined.

We have confirmed the above conjecture for all root systems of rank at most 8 , so the only open cases involve $C_{n}$ and $D_{n}$. Note that Brenti and Carnevale have explicit evaluations of the series $F_{J}\left(D_{n} ; q\right)$ for the cases with $|J|=1$, two cases with $|J|=2$, and some conjectured evaluations in a few cases with $|J| \leqslant 3$ (see Sections 5-6 of [2]); all are consistent with the above conjecture.

\section{REMARKS 7.5.}

(a) One way to prove that $F_{J}(\Phi ; q)$ factors into cyclotomic polynomials would be to show that it is a divisor of $F(\Phi ; q)$. (And empirically, it does not seem to matter whether we use the fine, coarse, or univariate gradings: for ranks up through 8, all three gradings share the same divisibility properties.) We will not attempt to formulate a comprehensive conjecture, but it appears that

(i) $F_{J}(\Phi ; \mathbf{q})$ is always a divisor of $F(\Phi ; \mathbf{q})$ under the fine grading for $A_{2 n}$ or $B_{n}$, and

(ii) among the classical cases, a sufficient condition for divisibility is that $\Gamma\left(\Phi_{0}\right) \cap W_{J}$ is trivial. Unfortunately, this condition is not sufficient for the exceptional cases.

(b) An even stronger divisibility condition would be for

$$
H_{J}(\Phi ; \mathbf{q}):=\frac{F(\Phi ; \mathbf{q})}{F_{J}(\Phi ; \mathbf{q}) F\left(\Phi_{J} ; \mathbf{q}\right)}
$$


to be a polynomial. If we assume $\Phi$ is irreducible and restrict to the coarse grading, so that $F\left(\Phi_{J} ; \mathbf{q}\right)$ is assured to be a divisor of $F(\Phi ; \mathbf{q})$ (Corollary 5.2 ), the only examples we have found where $F_{J}(\Phi ; \mathbf{q})$ divides $F(\Phi ; \mathbf{q})$ but $H_{J}(\Phi ; \mathbf{q})$ fails to be a polynomial involve $D_{n}$ for $n \geqslant 7$ and violate the condition in (a); i.e. $\Gamma\left(\Phi_{0}\right) \cap W_{J}$ is nontrivial.

REMARK 7.6. It would be natural to investigate the analogue of $F_{J}(\Phi ; \mathbf{q})$ in which the sum over $W^{J}$ is replaced with a sum over ${ }^{J} W$. However, these series do not appear to offer as much potential for factorization theorems. For example, when $\Phi=F_{4}$ or $E_{6}$, the univariate series factors into a product of cyclotomic polynomials only in the trivial case (i.e. $J=I$ ) and in the case $J=\varnothing$ (which coincides with the full series $F(\Phi ; q))$. Out of the 64 choices for $J$ available with $\Phi=C_{6}$ and 128 with $D_{7}$, only 6 in each case yield series that factor into cyclotomic polynomials.

\section{A UNIFIED PRESENTATION OF THE UNIVARIATE FACTORIZATION}

There is a uniform way to present the product formula for the univariate series $F(\Phi ; q)$ that involves data attached to a root system canonically associated to $\Phi$. To construct this second root system, we will need to work on the dual side with the co-root system $\Phi^{\vee}$.

Recall that the longest element $w_{0}$ of $W$ induces a diagram automorphism of $\Phi$ and $\Phi^{\vee}$ via $\beta \mapsto-w_{0} \beta$. Let $\sigma$ denote the induced map on the index set $I$ for the simple roots and co-roots, and $I / \sigma$ the set of $\sigma$-orbits on $I$. For each $\sigma$-orbit $J$, define

$$
\alpha_{J}^{\vee}:=\sum_{j \in J} \alpha_{j}^{\vee}=\left\{\begin{array}{cl}
\alpha_{j}^{\vee} & \text { if } j \in J \text { and } \sigma(j)=j, \\
\alpha_{j}^{\vee}+\alpha_{\sigma(j)}^{\vee} & \text { if } j \in J \text { and } \sigma(j) \neq j,
\end{array}\right.
$$

and let $s_{J}$ denote the longest element of $W_{J}$. It is easy to check that either

(i) $s_{J}$ is a simple reflection and $\alpha_{J}^{\vee} \in \Phi^{\vee}$ is simple, or

(ii) $s_{J}$ is a product of two commuting simple reflections and $\alpha_{J}^{\vee} \notin \Phi^{\vee}$, or

(iii) $s_{J}$ is the non-simple reflection in a copy of $A_{2}$ and $\alpha_{J}^{\vee} \in \Phi^{\vee}$ has height 2.

Note that (iii) occurs only if $\Phi^{\vee}$ has an irreducible component of type $A$ with even rank. Indeed, this is the only circumstance in which $\sigma$ reverses an edge of the diagram.

Since conjugation by $w_{0}$ acts as a diagram automorphism of any $\sigma$-stable parabolic subgroup $W_{J}$, it follows that the longest element of any such $W_{J}$ belongs to $Z\left(w_{0}\right)$, the centralizer of $w_{0}$. In particular, for each $\sigma$-orbit $J$, we have $s_{J} \in Z\left(w_{0}\right)$.

LEMMA 8.1. The centralizer $Z\left(w_{0}\right)$ is generated by $\left\{s_{J}: J \in I / \sigma\right\}$.

Proof. Consider that the $-w_{0}$-orbits $\left\{\beta^{\vee},-w_{0} \beta^{\vee}\right\}$ in $\Phi^{\vee}$ are permuted by $Z\left(w_{0}\right)$ and that each orbit consists of all positive or all negative co-roots. Therefore if $w \alpha_{j}^{\vee}<0$ for some $w \in Z\left(w_{0}\right)$, then the same is true for every index in the $\sigma$-orbit $J$ of $j$, and thus $w$ must be the longest element in its $W_{J}$-coset. Hence $\ell\left(w s_{J}\right)=\ell(w)-\ell\left(s_{J}\right)$ and it follows by induction that every member of $Z\left(w_{0}\right)$ has a length-additive expression as a product of elements chosen from $\left\{s_{J}: J \in I / \sigma\right\}$.

Let $V_{ \pm 1}$ denote the \pm 1 -eigenspaces for the action of $w_{0}$ on the ambient space $V$ that contains $\Phi$ and $\Phi^{\vee}$. Since $w_{0}$ is an involution, $V$ is the direct sum of these eigenspaces. Moreover, it is easy to see that $\left\{\alpha_{J}^{\vee}: J \in I / \sigma\right\}$ is a basis for $V_{-1}$; the parameter

$$
k:=|I / \sigma|=\operatorname{dim} V_{-1}
$$

will be important in what follows. Given that some of the generators $s_{J}$ may be products of two commuting reflections as in (ii), it is clear that $s_{J}$ need not be a reflection, and $Z\left(w_{0}\right)$ need not be a reflection subgroup of $W$. However as a centralizer, $Z\left(w_{0}\right)$ acts naturally on the eigenspaces of $w_{0}$, including $V_{-1}$. 
LEMMA 8.2. The centralizer $Z\left(w_{0}\right)$ acts faithfully on $V_{-1}$. Moreover, the generator $s_{J}$ acts on $V_{-1}$ as a reflection through the hyperplane orthogonal to $\alpha_{J}^{\vee}$.

Proof. In case the $\sigma$-orbit $J$ is of type (i) or (iii), $s_{J}$ acts on $V$ as a reflection through the hyperplane orthogonal to $\alpha_{J}^{\vee}$. Since $\alpha_{J}^{\vee} \in V_{-1}$, it restricts to a hyperplane reflection on $V_{-1}$ as well. If $J$ is of type (ii), so that $s_{J}$ is a product of two commuting simple reflections, say $s_{i} s_{j}$, then the -1-eigenspace of $s_{J}$ on $V$ is spanned by $\alpha_{i}^{\vee}$ and $\alpha_{j}^{\vee}$, but the intersection of this space with $V_{-1}$ is spanned by $\alpha_{J}^{\vee}=\alpha_{i}^{\vee}+\alpha_{j}^{\vee}$, so again it acts as a hyperplane reflection on $V_{-1}$. If some $w \in Z\left(w_{0}\right)$ acts trivially on $V_{-1}$, it cannot have any inversions (as a member of $W$ ); otherwise, the proof of Lemma 8.1 shows that it would have to invert the simple co-roots $\alpha_{j}^{\vee}$ for all $j$ in some $\sigma$-orbit $J$, and thus it would have to act nontrivially on $\alpha_{J}^{\vee} \in V_{-1}$. Hence the action is faithful.

Define $\Phi^{\vee} / \sigma$ to be the set of $-w_{0}$-orbit sums in $\Phi^{\vee}$; i.e.

$$
\Phi^{\vee} / \sigma:=\left\{\beta^{\vee}: w_{0} \beta^{\vee}=-\beta^{\vee}\right\} \cup\left\{\beta^{\vee}-w_{0} \beta^{\vee}: w_{0} \beta^{\vee} \neq-\beta^{\vee}\right\} .
$$

It is clear that $\Phi^{\vee} / \sigma$ is a union of $Z\left(w_{0}\right)$-orbits and that it contains each $\alpha_{J}^{\vee}$.

The following result is well-known; we include a proof for the sake of completeness.

Proposition 8.3. The orbit sums $\Phi^{\vee} / \sigma$ form a crystallographic root system in $V_{-1}$ of (full) rank $k$ with base $\left\{\alpha_{J}^{\vee}: J \in I / \sigma\right\}$. Hence $Z\left(w_{0}\right)$ is isomorphic to the Weyl group $W\left(\Phi^{\vee} / \sigma\right)$ via a map in which $s_{J}$ acts as the reflection corresponding to $\alpha_{J}^{\vee}$.

Proof. Let $\Sigma=\left\{\alpha_{J}^{\vee}: J \in I / \sigma\right\}$. By Lemma 8.2, it suffices to confirm that

(a) every member of $\Phi^{\vee} / \sigma$ is in the nonnegative or nonpositive $\mathbb{Z}$-span of $\Sigma$,

(b) every $Z\left(w_{0}\right)$-orbit in $\Phi^{\vee} / \sigma$ meets $\Sigma$, and

(c) no nontrivial rescaling of $\Phi^{\vee} / \sigma$ intersects $\Phi^{\vee} / \sigma$ (i.e. $\Phi^{\vee} / \sigma$ is reduced).

Given a $-w_{0}$-orbit $\mathcal{O}=\left\{\beta^{\vee},-w_{0} \beta^{\vee}\right\}$, we may exchange $\beta^{\vee}$ and $\mathcal{O}$ with $w_{0} \beta^{\vee}$ and $-\mathcal{O}$ if necessary so that $\beta^{\vee}>0$. In that case, $\beta^{\vee}$ and $-w_{0} \beta^{\vee}$ must have nonnegative integer coordinates with respect to the simple roots of $\Phi^{\vee}$, so the same is true for the $\Sigma$-coordinates of the orbit sum, proving (a).

Since $w_{0} \in Z\left(w_{0}\right)$ inverts all co-roots, there must be an element $w \in Z\left(w_{0}\right)$ and a generator $s_{J}$ such that $w \mathcal{O}$ is a positive orbit and $s_{J} w \mathcal{O}$ is negative. However, $s_{J}$ inverts only the roots in the subsystem $\Phi_{J}^{\vee}$; in most cases, this forces $w \mathcal{O}$ to be $\left\{\alpha_{j}^{\vee}: j \in J\right\}$ and the corresponding orbit sum to be $\alpha_{J}^{\vee}$. The one exception occurs when the $\sigma$-orbit $J$ is of type (iii), in which cases there are two positive $-w_{0}$-orbits in $\Phi_{J}^{\vee}$ : one singleton and one doubleton. However they both have sum $\alpha_{J}^{\vee}$, so (b) follows either way. (This also shows that the union in (10) need not be disjoint.)

Finally, suppose $\Phi^{\vee} / \sigma$ and $k \Phi^{\vee} / \sigma$ had a nontrivial intersection for some scalar $k>1$. In that case, (b) would force some $Z\left(w_{0}\right)$-orbit in $\Phi^{\vee} / \sigma$ to have $\Sigma$-coordinates in $k \mathbb{Z}$, contradicting the fact that the orbit must include some $\alpha_{J}^{\vee}$.

Now let $L_{0}^{\vee}$ denote the kernel of the parity morphism $\mathbb{Z} \Phi^{\vee} \rightarrow \mathbb{Z} / 2 \mathbb{Z}$ in which every simple co-root is odd; this is the co-root counterpart to the lattice $L_{0}$ we introduced in (1). Having constructed a root system $\Phi^{\vee} / \sigma$ contained in $\mathbb{Z} \Phi^{\vee}$, it follows that

$$
\left(\Phi^{\vee} / \sigma\right)_{0}:=L_{0}^{\vee} \cap\left(\Phi^{\vee} / \sigma\right)
$$

is a root subsystem of $\Phi^{\vee} / \sigma$. Note that if $w_{0}$ acts as -1 on $\Phi^{\vee}$, the involution $\sigma$ is trivial, $\Phi^{\vee} / \sigma=\Phi^{\vee}$, and $\left(\Phi^{\vee} / \sigma\right)_{0}=L_{0}^{\vee} \cap \Phi^{\vee}$ is the even-height root subsystem of $\Phi^{\vee}$.

It is important to note that in general, some of the simple roots of $\Phi^{\vee} / \sigma$ may be "even" (i.e. members of $\left.L_{0}^{\vee}\right)$ and thus part of $\left(\Phi^{\vee} / \sigma\right)_{0}$. That is, the parity that $L_{0}^{\vee}$ induces on $\Phi^{\vee} / \sigma$ need not be the one that declares all of its simple roots to be odd. 
EXAMPLE 8.4. Consider $\Phi=A_{n}$, with the diagram numbered $1-2-\cdots-n$. Here, the action of $\sigma$ is such that $\sigma(i)=n+1-i$. If $n=2 r$, the simple roots of $\Phi^{\vee} / \sigma$ are

$$
\alpha_{r}^{\vee}+\alpha_{r+1}^{\vee}, \quad \alpha_{r-1}^{\vee}+\alpha_{r+2}^{\vee}, \ldots, \quad \alpha_{1}^{\vee}+\alpha_{2 r}^{\vee} .
$$

The first one is of type (iii) while the others are of type (ii) and thus longer, and it is not hard to check that the root system they generate is isomorphic to $B_{r}$. Moreover, all of these roots are in $L_{0}^{\vee}$, so we have $\Phi^{\vee} / \sigma=\left(\Phi^{\vee} / \sigma\right)_{0} \cong B_{r}$. On the other hand, if $n=2 r-1$, the simple roots of $\Phi^{\vee} / \sigma$ are

$$
\alpha_{r}^{\vee}, \quad \alpha_{r-1}^{\vee}+\alpha_{r+1}^{\vee}, \ldots, \quad \alpha_{1}^{\vee}+\alpha_{2 r-1}^{\vee} .
$$

The first one is again short, but it is of type (i) and has odd height in $\Phi^{\vee}$. Thus $\Phi^{\vee} / \sigma \cong B_{r}$, but in this case $\left(\Phi^{\vee} / \sigma\right)_{0} \cong D_{r}$ contains only the long roots. (In the somewhat degenerate case $\Phi=A_{1}$, one should recognize that $\left(\Phi^{\vee} / \sigma\right)_{0} \cong D_{1}$ is an empty root system.)

\begin{tabular}{|c|c|c|c|c|}
\hline$\Phi$ & $\Phi_{0}$ & $\Phi^{\vee} / \sigma$ & $\left(\Phi^{\vee} / \sigma\right)_{0}$ & $k$ \\
\hline$A_{2 n-1}$ & $A_{n-1} \oplus A_{n-1}$ & $B_{n}$ & $D_{n}$ & $n$ \\
$A_{2 n}$ & $A_{n} \oplus A_{n-1}$ & $B_{n}$ & $B_{n}$ & $n$ \\
$B_{2 n}$ & $B_{n} \oplus D_{n}$ & $C_{2 n}$ & $A_{2 n-1}$ & $2 n$ \\
$B_{2 n+1}$ & $B_{n} \oplus D_{n+1}$ & $C_{2 n+1}$ & $A_{2 n}$ & $2 n+1$ \\
$C_{2 n}$ & $A_{2 n-1}$ & $B_{2 n}$ & $B_{n} \oplus D_{n}$ & $2 n$ \\
$C_{2 n+1}$ & $A_{2 n}$ & $B_{2 n+1}$ & $B_{n} \oplus D_{n+1}$ & $2 n+1$ \\
$D_{2 n}$ & $D_{n} \oplus D_{n}$ & $D_{2 n}$ & $D_{n} \oplus D_{n}$ & $2 n$ \\
$D_{2 n+1}$ & $D_{n} \oplus D_{n+1}$ & $C_{2 n}$ & $C_{n} \oplus C_{n}$ & $2 n$ \\
$E_{6}$ & $A_{5} \oplus A_{1}$ & $F_{4}$ & $B_{4}$ & 4 \\
$E_{7}$ & $A_{7}$ & $E_{7}$ & $A_{7}$ & 7 \\
$E_{8}$ & $D_{8}$ & $E_{8}$ & $D_{8}$ & 8 \\
$F_{4}$ & $C_{3} \oplus A_{1}$ & $F_{4}$ & $C_{3} \oplus A_{1}$ & 4 \\
$G_{2}$ & $A_{1} \oplus A_{1}$ & $G_{2}$ & $A_{1} \oplus A_{1}$ & 2 \\
\hline
\end{tabular}

TABLE 3. Listings of $\Phi_{0}, \Phi^{\vee} / \sigma,\left(\Phi^{\vee} / \sigma\right)_{0}$, and $k=\operatorname{dim} V_{-1}$.

In Table 3, we list the isomorphism classes of $\Phi_{0}, \Phi^{\vee} / \sigma$, and $\left(\Phi^{\vee} / \sigma\right)_{0}$ as well as the parameter $k=\operatorname{dim} V_{-1}$ (the rank of $\left.\Phi^{\vee} / \sigma\right)$ for each irreducible $\Phi$.

To place the following result in its proper context, recall that for any finite reflection group action, such as $W$ acting on $V$, there is a canonically associated multiset of positive integers $d_{1}, \ldots, d_{m}$ (where $m=\operatorname{dim} V$ ). These are the degrees of a homogeneous set of free generators for the $W$-invariants in the symmetric algebra $S(V)$. (The existence of such generators is Chevalley's Theorem; e.g. see [5, §3.5].)

THEOREM 8.5. If $a_{1}, \ldots, a_{k}$ are the degrees of the free generating invariants for the action of $W\left(\left(\Phi^{\vee} / \sigma\right)_{0}\right)$ on $S\left(V_{-1}\right)$, then

$$
F(\Phi ; q)=\prod_{i=1}^{k}\left(1-q^{a_{i}}\right)
$$

Proof. It suffices to prove this when $\Phi$ is irreducible. In such cases, we may obtain the univariate series $F(\Phi ; q)$ by specializing Theorem 6.1 . On the product side, one may use Table 3 to determine the degrees $a_{1}, \ldots, a_{k}$. Since the components of $\left(\Phi^{\vee} / \sigma\right)_{0}$ are 
always of classical type, one only needs to know that the degrees of the generating invariants for the action on the span of such a component are as follows:

$$
\begin{aligned}
A_{n} & : 2,3, \ldots, n+1, \\
B_{n}, C_{n}: & 2,4,6, \ldots, 2 n, \\
D_{n}: & n, 2,4,6, \ldots, 2 n-2 .
\end{aligned}
$$

It should be noted that the rank $r$ of $\left(\Phi^{\vee} / \sigma\right)_{0}$ may be less than $k$, in which case the action of $W\left(\left(\Phi^{\vee} / \sigma\right)_{0}\right)$ on the span of $\left(\Phi^{\vee} / \sigma\right)_{0}$ accounts for only $r$ of the $k$ generating invariants in $S\left(V_{-1}\right)$. In such cases, the remaining $k-r$ generators all have degree 1 . (This happens when $\Phi \cong A_{1}, C_{2}$, or $B_{n}$.)

\section{REMARKS 8.6.}

(a) Let $G=W\left(\left(\Phi^{\vee} / \sigma\right)_{0}\right)$. The Hilbert series for the $G$-invariants is

$$
H\left(S\left(V_{-1}\right)^{G} ; q\right)=\prod_{i=1}^{k} \frac{1}{1-q^{a_{i}}},
$$

so an equivalent formulation of Theorem 8.5 is the identity

$$
F(\Phi ; q) H\left(S\left(V_{-1}\right)^{G} ; q\right)=1 .
$$

(b) The degrees of the generating invariants also appear in Chevalley's formula for the (untwisted) Poincaré series $P(W ; q)=\sum_{w \in W} q^{\ell(w)}$ for any Weyl group $W$ (e.g. see $[5, \S 3.15])$. Thus another way to formulate Theorem 8.5 is the identity

$$
\frac{F(\Phi ; q)}{(1-q)^{k}}=P\left(W\left(\left(\Phi^{\vee} / \sigma\right)_{0}\right) ; q\right) .
$$

It should be stressed that the length function here is the one intrinsic to the Weyl group of the root system $\left(\Phi^{\vee} / \sigma\right)_{0}$. It is not the same as the length functions for the Weyl groups of $\Phi^{\vee} / \sigma$ or $\Phi$ (although the proof of Lemma 8.1 shows that the latter two are related). Note also that the factor $(1-q)^{-k}$ and the right hand side of (11) may be interpreted as the Hilbert series for $S\left(V_{-1}\right)$ and its associated co-invariant algebra, respectively.

(c) It would be interesting to develop a multivariate refinement of Theorem 8.5 for either the coarse or fine gradings of $F(\Phi ; \mathbf{q})$. In this context it should be noted that Macdonald has given a coarse refinement of $P(W ; q)$ for any Weyl group $W[9]$.

\section{REFERENCES}

[1] N. Bourbaki, Groupes et algèbres de Lie, Chp. IV-VI, Masson, Paris, 1981.

[2] F. Brenti and A. Carnevale, Odd length for even hyperoctahedral groups and signed generating functions, Discrete Math. 340 (2017), no. 12, 2822-2833.

[3] _ Odd length in Weyl groups, https://arxiv.org/abs/1709.03320, 2017.

[4] , Proof of a conjecture of Klopsch-Voll on Weyl groups of type A, Trans. Amer. Math. Soc. 369 (2017), 7531-7547.

[5] J. E. Humphreys, Reflection groups and Coxeter groups, Cambridge Univ. Press, Cambridge, 1990.

[6] B. Klopsch and C. Voll, Igusa-type functions associated to finite formed spaces and their functional equations, Trans. Amer. Math. Soc. 361 (2009), no. 8, 4405-4436.

[7] Anthony W. Knapp, Lie groups beyond an introduction, Progress in Mathematics, vol. 140, Birkhäuser, Boston, MA, 1996.

[8] A. Landesman, Proof of Stasinski and Voll's hyperoctahedral group conjecture, Australas. J. Combin. 71 (2018), no. 2, 196-240.

[9] I. G. Macdonald, The Poincaré series of a Coxeter group, Math. Ann. 199 (1972), no. 2, 161174 . 
John R. Stembridge

[10] A. Stasinski and C. Voll, A new statistic on the hyperoctahedral groups, Electron. J. Combin. 20 (2013), no. 3, P50 (23 pages).

[11] _ Representation zeta functions of nilpotent groups and generating functions for Weyl groups of type B, Amer. J. Math. 136 (2014), no. 2, 501-550.

John R. Stembridge, Dept. of Mathematics, University of Michigan, Ann Arbor, Michigan 481091043, USA

E-mail : jrs@umich.edu 